\title{
Prospects for radio detection of ultra-high energy cosmic rays and neutrinos
}

\author{
H. Falcke ${ }^{\mathrm{a}}$, P. Gorham ${ }^{\mathrm{b}}$, R.J. Protheroe ${ }^{\mathrm{c}}$ \\ a ASTRON, P.O. Box 27990 AA Dwingeloo, The Netherlands, falcke@astron.nl \\ ${ }^{\mathrm{b}}$ Department of Physics \& Astronomy, Univ. of Hawaii at Manoa, Honolulu, HI, 96822, USA, \\ gorham@phys.hawaii.edu
}

cDepartment of Physics, University of Adelaide, SA 5006, Australia, rprother@physics.adelaide.edu.au

The origin and nature of the highest energy cosmic ray events is currently the subject of intense investigation by giant air shower arrays and fluorescent detectors. These particles reach energies well beyond what can be achieved in ground-based particle accelerators and hence they are fundamental probes for particle physics as well as astrophysics. One of the main topics today focuses on the high energy end of the spectrum and the potential for the production of high-energy neutrinos. Above about $10^{20} \mathrm{eV}$ cosmic rays from extragalactic sources are expected to be severely attenuated by pion photoproduction interactions with photons of the cosmic microwave background. Investigating the shape of the cosmic ray spectrum near this predicted cut-off will be very important. In addition, a significant high-energy neutrino background is naturally expected as part of the pion decay chain which also contains much information.

Because of the scarcity of these high-energy particles, larger and larger ground-based detectors have been built. The new generation of digital radio telescopes may play an important role in this, if properly designed. Radio detection of cosmic ray showers has a long history but was abandoned in the 1970's. Recent experimental developments together with sophisticated air shower simulations incorporating radio emission give a clearer understanding of the relationship between the air shower parameters and the radio signal, and have led to resurgence in its use. Observations of air showers by the SKA could, because of its large collecting area, contribute significantly to measuring the cosmic ray spectrum at the highest energies. Because of the large surface area of the moon, and the expected excellent angular resolution of the SKA, using the SKA to detect radio Cherenkov emission from neutrino-induced cascades in lunar regolith will be potentially the most important technique for investigating cosmic ray origin at energies above the photoproduction cut-off.

\section{Observational and theoretical motiva- tion}

Understanding the origin of the ultra-high energy cosmic rays (UHECR), the highest energy particles observed in nature, is of great importance as it may impact our understanding of particle physics, fundamental cosmology, and extremely energetic phenomena in the Universe. The energy spectrum of UHECR extends up to at least $10^{11} \mathrm{GeV}$, and in the rest frame of a UHECR proton, photons of the $2.73 \mathrm{~K}$ cosmic microwave background radiation (CMBR) are strongly blueshifted to gamma-ray energies. The threshold for Bethe-Heitler pair production and pion photopro- duction by UHECR protons on the CMBR are close to $2 \times 10^{8} \mathrm{GeV}$ and $2 \times 10^{10} \mathrm{GeV}$, such that protons at $3 \times 10^{10} \mathrm{GeV}$ and $3 \times 10^{11} \mathrm{GeV}$ typically lose a large fraction of their energy in a time of $1 \mathrm{Gpc} / \mathrm{c}\left(3 \times 10^{9} \mathrm{y}\right)$ and $10 \mathrm{Mpc} / \mathrm{c}\left(3 \times 10^{7} \mathrm{y}\right)$, respectively. This would imply that sources of ultra-high energy cosmic rays would have to be close if the particles themselves behave as predicted. The importance of pion photoproduction on the CMBR was first noted by Greisen (1966) and Zatsepin \& Kuzmin (1966) and the cut-off they predicted at $\sim 10^{11} \mathrm{GeV}$ is referred to as the "GZK cut-off".

Yet, until today neither the nature of the par- 
ticles nor of their accelerators has been revealed. It is well established that in some astrophysical magnetized plasma regions particles (leptons) are accelerated, but whether and where this holds for UHECR is unclear. Some basic constraints can nonetheless be given. Cosmic ray acceleration sites must be large enough to contain the gyroradius of the accelerated particles, as well as having scattering centres with appropriate velocities. In addition, the acceleration must be sufficiently rapid that high energies can be achieved in an accelerator's lifetime, and that energy losses by pion photoproduction and synchrotron radiation do not cut off the spectrum too soon (Hillas 1984). It is currently unknown whether the UHECR are Galactic or extragalactic in origin. Composition measurements are also important because if UHECR are observed to include nuclei other than protons then these must be from Galactic, or very nearby extragalactic acceleration sources to avoid photodisintegration (see e.g. Yamamoto et al. 2004). However, the promising extragalactic source candidates for UHECR above $10^{11} \mathrm{GeV}$ are typically at distances too far for UHECR to reach us unaffected by interactions with the CMBR. This is the basic dilemma we are faced with today.

\subsection{UHECR observations}

Below the GZK cut-off UHECR may, to some extent, point back to their sources depending on the structure and strength of the magnetic field between the sources and our Galaxy. No statistically compelling anisotropy has been detected in the UHE CR. The energy spectrum of UHECR detected by AGASA is shown in Fig. 1 There are two main problems at present: the flux of UHECR is so low that few events have been detected for reliable conclusions concerning the presence or absence of a GZK cut-off or any anisotropy, and in the case of a GZK cut-off spectral information above the cut-off would be lost. New experiments such as HiRes and the $3000 \mathrm{~km}^{2}$ Peirre Auger Observatory will help to address the the first issue and, because of its huge area, use of the SKA may also help here by direct radio detection of UHECR air showers. The second problem, that of loss of spectral information above the

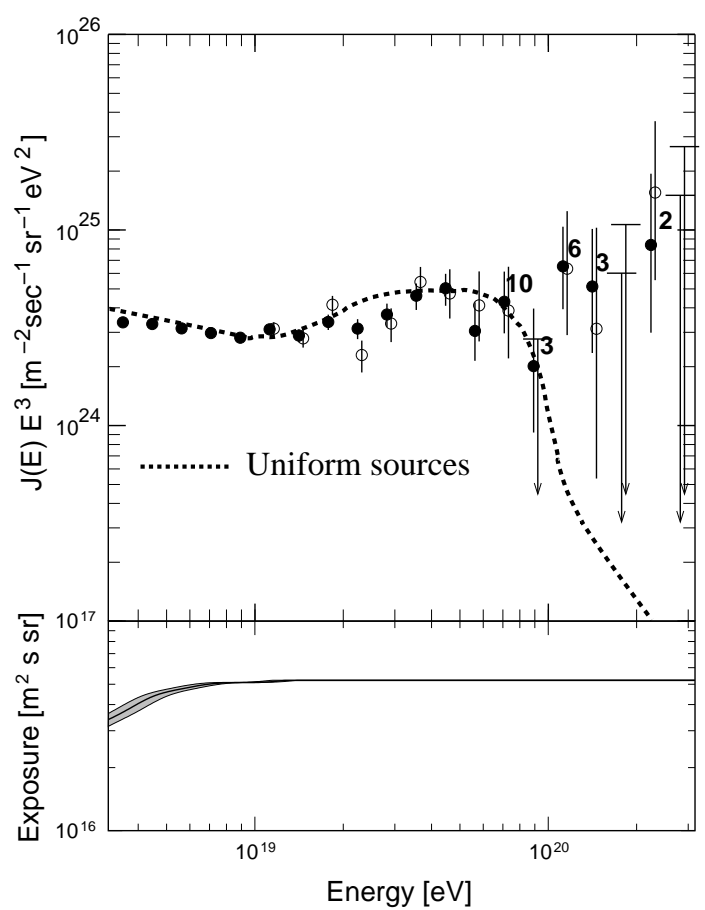

Figure 1. Spectrum of UHECR detected by AGASA. Numbers attached to points show the number of events in each energy bin. (From Takeda et al. 2003.)

GZK cut-off, is best explored through UHE neutrino astronomy and again the use of the SKA, either to detect neutrino-induced air showers or radio Cherenkov bursts from electromagnetic cascades in lunar regolith initiated by interactions of UHE neutrinos, has the potential to greatly add to our knowledge of the the origin of the highest particles in nature.

\subsection{The GZK problem}

Due to interactions with the CMBR, there is expected to be a spectral downturn, the GZK cut-off, for particles which have travelled more than a few tens of Mpc. Figure 2 shows the distribution in energy as a function of distance travelled for UHECR protons with initial ener- 


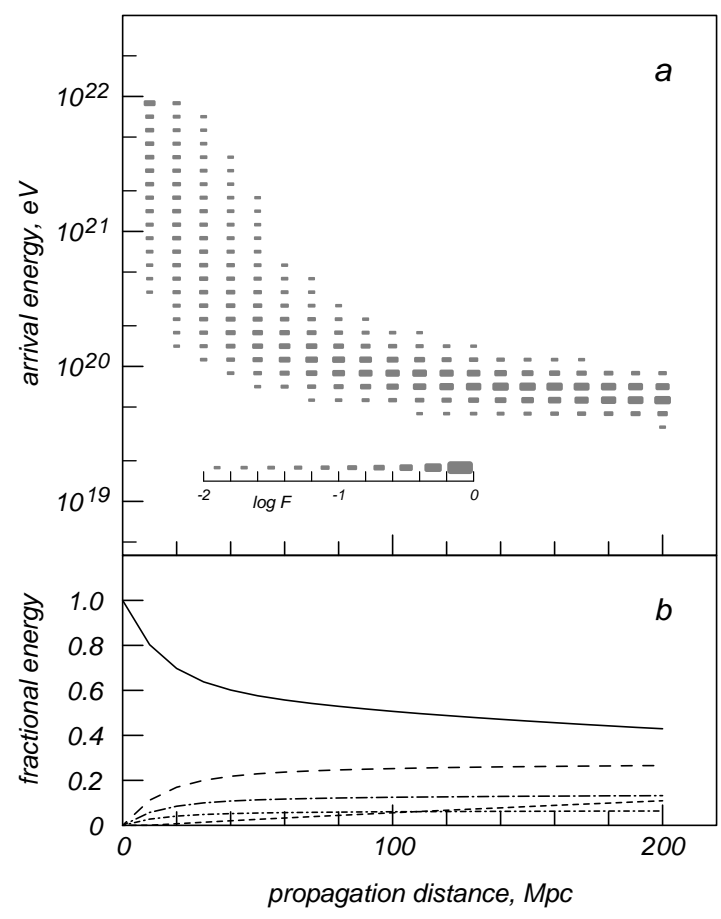

Figure 2. (a) Arrival energy distribution for protons injected with energy between $10^{21.9}$ and $10^{22} \mathrm{eV}$ after propagation on $10,20, \ldots 200$ Mpc. (b) Fractional energy contained in nucleons (solid line), $\gamma$-rays from photoproduction (long dashes) and $\mathrm{BH}$ pair production (short dashes) for protons injected with a $E^{-2}$ power law spectrum with an exponential cutoff at $10^{21.5} \mathrm{eV}$. The dash-dot lines show the fractional energy in muon (long) and electron (short) neutrinos and antineutrinos. (From Stanev at al. 2000.)

gies close to $10^{22} \mathrm{eV}$. As can be seen, after $80 \mathrm{Mpc}$ no protons have energies above $3 \times 10^{20} \mathrm{eV}$. Of course, UHECR protons are also deflected by extragalactic magnetic fields, and so any source of $3 \times 10^{20} \mathrm{eV}$ UHECR would need to be much nearer than $80 \mathrm{Mpc}$. However, several experiments have reported CR events with energies above $10^{20} \mathrm{eV}$ with the highest energy event having $3 \times 10^{20} \mathrm{eV}$
(Bird et al.1995). Very recent data from the two largest aperture high energy cosmic ray detectors are contradictory: AGASA (Takeda et al. 2003) observes no GZK cut-off while HiRes (Abbasi et al. 2004) observes a cut-off consistent with that expected. A systematic over-estimation of energy of about $25 \%$ by AGASA or under-estimation of energy of about $25 \%$ by HiRes could account the discrepancy (Abbasi et al. 2004), but the continuation of the UHECR spectrum to energies well above $10^{20} \mathrm{eV}$ is now far from certain. Future measurements with Auger (Auger Collaboration 2001) should resolve this question. Whether or not the spectrum does extend well beyond $10^{20} \mathrm{eV}$, determining the origin of these particles could have important implications for astrophysics, cosmology and particle physics.

\subsection{The acceleration problem}

By plotting magnetic field vs. size of various astrophysical objects (Fig. 31), Hillas (1984) identified possible sites of acceleration of UHECR based on whether the putative source could contain the gyroradius of the accelerated particles, and on the likely velocity of scattering centres in these sites. Following Hillas (1984) one finds that possible sites included neutron stars $\left(10^{7}-\right.$ $10^{13} \mathrm{G}$ ), gamma ray bursts and active galactic nuclei $\left(10^{3}-10^{4} \mathrm{G}\right)$, and lobes of giant radio galaxies and galaxy clusters $\left(10^{-7}-10^{-5} \mathrm{G}\right)$. This identification of possible sources does not take account of energy losses (synchrotron) and interactions (Bethe-Heitler and pion photoproduction) which can cut off the spectrum, and so apply an additional constraint which we discuss below (in his original paper Hillas (1984) also used this additional constraint to narrow the field of possible sources).

For particle acceleration by electric fields induced by the motion of magnetic fields $B$ (including those at astrophysical shocks), the maximum rate of momentum gain by relativistic particles of charge $Z e$ can be written (in SI units) $(d p / d t)_{\text {acc }}=\xi(p) Z e c B$ where $\xi(p)<1$ is the acceleration rate parameter and depends on the details of the acceleration mechanism (see the review by Jones \& Ellison 1991, on the plasma physics of shock acceleration, which also includes 


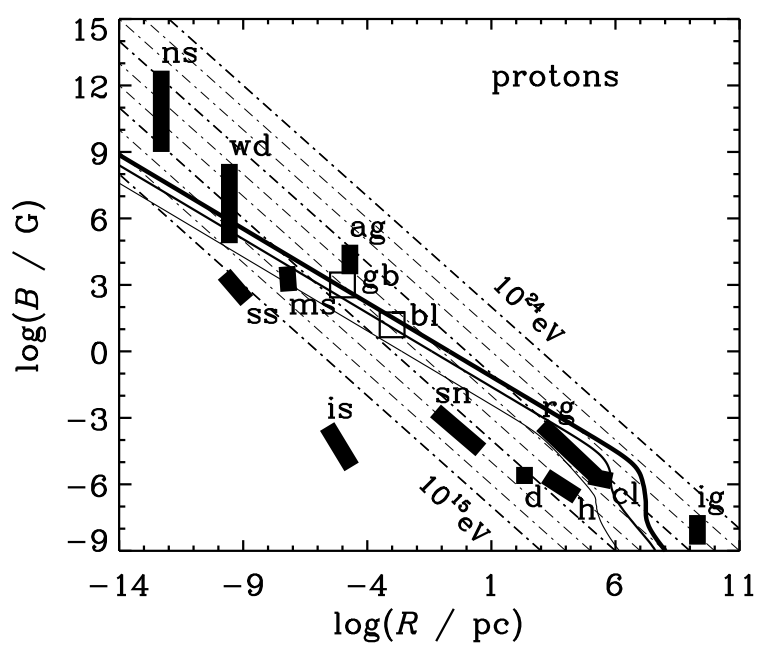

Figure 3. "Hillas plot" showing (chain curves) magnetic field vs. gyroradius for proton momenta $10^{15}, 10^{16}, \ldots, 10^{24} \mathrm{eV} / \mathrm{c}$. The solid curves bound the parameter space of accelerated particles for a given acceleration rate parameter (see text). Typical size and magnetic field of possible acceleration sites (taken from Hillas 1984) are shown for neutron stars (ns), white dwarfs (wd), sunspots (ss), magnetic stars (ms), AGN (ag), interstellar space (is), supernova remnants (sn), radio galaxy lobes (rg), galactic disk (d) and halo (h), clusters of galaxies (cl) and intergalactic medium (ig). Typical jet-frame parameters of the synchrotron proton blazar model (Müecke et al. 2003) and gamma ray burst model (Pelletier \& Kersate 2000) are indicated by the open squares labelled "bl" and and "gb". (From Protheroe 2004.)

a brief historical review and refers to early work). To estimate cut-off momenta (or energies), one needs the acceleration rate. The following values for the acceleration rate parameter have been suggested: maximum possible acceleration rate $\xi\left(p_{\text {cut }}\right)=1$, plausible acceleration at perpendicular shock with speed $0.1 c, \xi\left(p_{\text {cut }}\right) \approx 0.04$, and plausible acceleration at parallel shock with speed $0.1 c$,

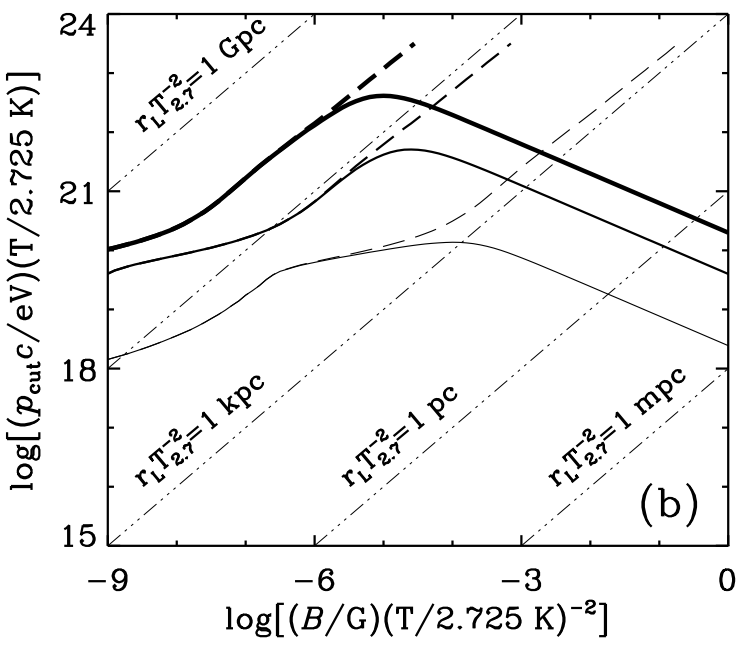

Figure 4. Maximum energy as a function of magnetic field of protons for maximum possible acceleration rate $\xi=1$ (upper solid curve), $\xi=0.04$ (middle solid curve), $\xi=1.5 \times 10^{-4}$ (lower solid curve). Dashed curves are limits from BetheHeitler pair production and pion photoproduction only (solid curves include synchrotron loss). Dotdot-dot-dash curves are lines of constant Larmor radius as labelled. (From Protheroe 2004.)

$\xi\left(p_{\text {cut }}\right) \approx 1.5 \times 10^{-4}$ (Protheroe 2000). Based on the total momentum loss rate for Bethe-Heitler pair production and pion photoproduction on the CMBR, synchrotron losses and redshifting the proton cut-off momentum is plotted in Fig. 4 as a function of magnetic field for three adopted $\xi$ values (chain lines are for constant Larmor radius as labelled). This plot clearly shows that to accelerate protons to $\sim 10^{20} \mathrm{eV}$ large regions of relatively low magnetic field $\sim 10^{-7}-10^{-3} \mathrm{G}$ are needed, apparently ruling out high magnetic field regions for the origin of UHECR (see also Medvedev 2003). One sees that, in principle, protons can be accelerated up to $\sim 5 \times 10^{22} \mathrm{eV}$ in Mpc scale region with $\sim 10^{-5} \mathrm{G}$.

Returning to the Hillas plot (Fig. 3), constraints have been added corresponding to the 
three curves in Fig. 4 and the chain lines give constant proton energy values as indicated. Sources to the right of the solid curves are excluded; a possible exception to this is in the case of relativistically beamed sources (e.g. for AGN see Protheroe et al. 2003, and for GRB see Pelletier \& Kersate 2000) where neutrons emitted along the direction of relativistic motion can be Doppler boosted significantly in energy. Another possible exception is the case of so called "one-shot" mechanisms (e.g. Haswell et al . 1992, Sorrell 1987) where a particle is accelerated by an electric field along a nearly straight path which is essentially parallel to the magnetic field such that curvature and synchrotron losses are negligible. Suggested sites for this include polarization electric fields arising in plasmoids injected into a neutron star's magnetosphere (Litwin \& Rosner 2001) and magnetic re-connection in the magnetosphere of accretion induced collapse pulsars (de Gouvela Dal Pino \& Lazarian 2001). Another possibility is plasma wakefield acceleration, i.e. acceleration by collective plasma waves, possibly in the atmosphere of a GRB, or "surf-riding" in the approximately forcefree fields of the relativistic wind of a newly born magnetar (Arons 2003). In these cases it is unclear whether the requirements of negligible radiation losses can be met.

Alternative scenarios for UHECR origin include emission and decay of massive particles ("X-particles") by topological defects (TD) or decay of massive primordial particles. Because of the resulting flat spectrum of particles (including neutrinos, gamma-rays and protons) extending possibly up to GUT (grand unified theory) scale energies, topological defect models have been invoked to try to explain the UHECR. Propagation of the spectra of all particle species over cosmological distances is necessary to predict the cosmic ray and gamma-ray spectra expected at Earth. In most cases this results in excessive gammaray fluxes at $\mathrm{GeV}$ energies in addition to cosmic rays. Massive relic particles on the other hand, would cluster in galaxy halos, including that of our Galaxy, and may give rise to anisotropic cosmic ray signals at ultra high energies. One possibility proposed for getting around this is if an extragalactic source emits a very high luminos- ity in UHE neutrinos, some of which interact with relic neutrinos gravitationally bound to our galaxy producing "Z-bursts" which generate the events observed above the expected GZK cut-off. (see Protheroe \& Clay 2004 for a recent review of UHECR.)

\subsection{Neutrino signatures}

One way of getting information about acceleration sources of UHECR is through the spectral shape near acceleration cut-off. One of the present authors (Protheroe 2004) has recently shown that in the case of protons the spectrum can actually be quite sensitive to the astrophysical acceleration environment. Despite the fact that for extragalactic UHECR almost all spectral information above the GZK cut-off is lost, significant information is preserved in the spectrum of neutrinos produced as a result of pion photoproduction interactions during propagation (Protheroe 2004). Furthermore, the spectrum of these GZK neutrinos differs significantly from that in Z-burst and topological defect (TD) scenarios, and of course the neutrinos are not deflected by magnetic fields and so should point back to where they were produced. Hence, UHE neutrino astronomy will be able provide much needed clues to the origin of the UHECR.

The spectra of protons and neutrinos escaping from an acceleration source and after propagation for $100 \mathrm{Mpc} / \mathrm{c}$ is shown in Fig. [5for various possible acceleration environments represented by the spectrum of magnetic turbulence present (powerlaw dependence of acceleration rate), the average magnetic field, its alignment, speed of scattering centres (acceleration rate and maximum energy), and the size of the acceleration region (decay or escape of photoproduced neutrons). As can be seen, while there is little difference in the spectrum of UHECR after propagation over $100 \mathrm{Mpc}$, much information is preserved in the spectrum of UHE neutrinos ("GZK neutrinos") produced during propagation as the UHECR flux is eroded by the GZK-cutoff effect. Of course, for very distant sources UHECR would not be expected to be observed from directions of sources. Indeed, few if any may arrive at all because of difficulty in reaching Earth through extragalactic magnetic 


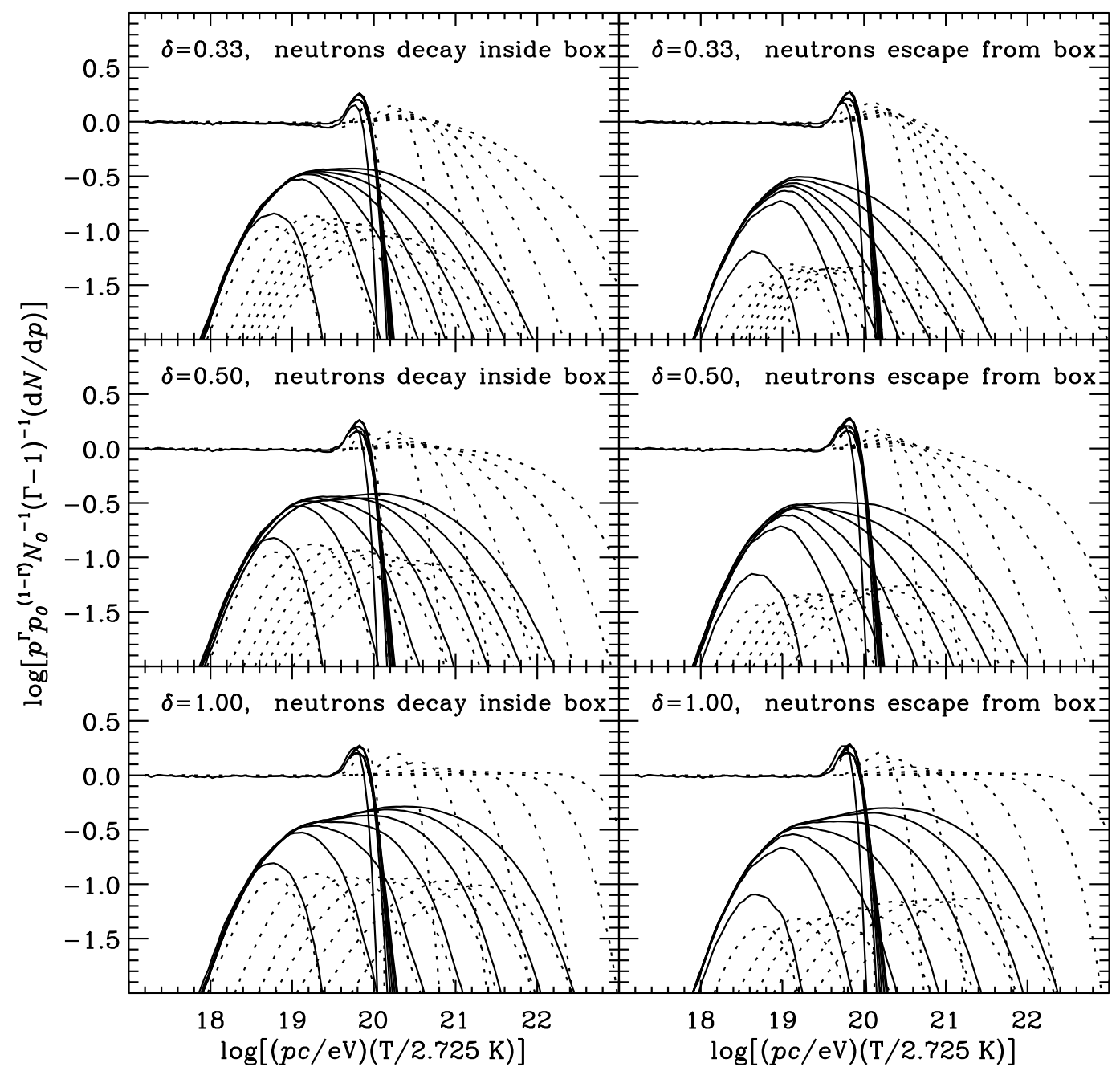

Figure 5. Spectra of protons and neutrinos (all flavours) escaping from the acceleration region (dotted curves) and after propagation for time $100 \mathrm{Mpc} / \mathrm{c}$ (solid curves) for $p_{\text {cut }} c=10^{20}$ (leftmost curves), $10^{20.5}$, $\ldots, 10^{23} \mathrm{eV}$ (rightmost curves); $\Gamma=2$. (From Protheroe 2004.)

fields, whereas UHE neutrinos will arrive essentially undeflected. A very sensitive UHE neutrino telescope may therefore observe neutrinos from extragalactic UHECR sources. The diffuse GZK neutrino background can actually be quite large if the UHECR sources evolve strongly with redshift (Engel et al. 2001). Nevertheless, huge collecting areas will be required for the detection of UHE neutrinos, and it is here that the SKA through direct detection of neutrino-induced air showers and, perhaps more importantly, through the detection of Cherenkov radio transients from neutrino-induced showers in lunar regolith may make a major contribution to understanding the 
origin of the UHECR.

\section{Detection of high energy particles: his- torical background}

There is clearly strong theoretical and phenomenological motivation to detect both the presumably hadronic cosmic rays and associated neutrinos at $\mathrm{EeV}$ to $\mathrm{ZeV}$ energies. The difficulty arises from the extremely low fluxes present-for the highest energy cosmic rays at or above the $\sim$ $6 \times 10^{19} \mathrm{eV}$ GZK cutoff, one can expect of order a few per $\mathrm{km}^{2}$ per century at most. The associated $\mathrm{EeV}$ neutrino fluxes are, in the most optimistic scenarios, perhaps 1-2 orders of magnitude larger than this, but their detection efficiency is at most $\sim 1 \%$ per cubic $\mathrm{km}$ of water-equivalent material, and thus the neutrino rates are abysmally low in all existing and most planned detectors (though a recently approved NASA long-duration balloon experiment, ANITA (Barwick et al. 2003), may get an early, low-resolution view of these fluxes).

\subsection{Giant air shower detectors.}

Since the early 1960's through the mid-1980's the highest energy cosmic ray detectors were exclusively large ground arrays of scintillators or Cherenkov counters making direct detection of secondary particles, mainly electrons, gammarays, and muons within the confines of the air shower itself as it impacts the ground (see Nagano and Watson 2000 for a review and references to major air shower detectors). At the highest energies, air shower detectors gain much of their collecting aperture by capturing the edges of showers whose cores fall outside their fiducial array boundaries, sometimes by many hundreds of meters. Thus the shower energy must be estimated by parametric models for the particle density at the shower periphery - a technique which has undergone much evolution throughout the history of giant air shower detection, and still retains much controversy in the details of its application even today.

In the mid-1980's the first Nitrogen air fluorescence (N2fl) detector, the Fly's Eye, came online. Since that time, both the Fly's Eye and the follow-on High Resolution Fly's Eye (HiRes) have become competitive with the air shower ground arrays in their detection efficiency and aperture, and HiRes now has the largest exposure and data sample of any detector to date.

The N2fl technique is very different than that of ground array detection, since the detectors do not require direct intersection with any portion of the air shower particles, but rather detect the secondary incoherent radiation from deexcitation of Nitrogen heated by the passage of the shower. Such emission may be seen by optical telescopes of several $\mathrm{m}^{2}$ aperture out to tens of $\mathrm{km}$ distance from the shower itself, and thus a small installation of modest, low-optical quality (e.g., searchlight-style) reflectors can, by viewing a good fraction of the surrounding sky, create an effective air shower collecting aperture of several thousand $\mathrm{km}^{2}$ sr. The only drawbacks to this technique are its sensitivity to atmospheric attenuation in the near ultraviolet (where the nitrogen emission lies), and its requirement for complete darkness and clear weather. These constraints lead to a low net long-term duty cycle of less than $10 \%$, compared to the $\sim 100 \%$ duty cycle of a ground array.

\subsection{Cosmic ray air shower radio detection}

Interest in radio techniques for giant air shower detection stemmed originally from the suggestion by Askaryan (1962) that any electromagnetic cascade in a dielectric material (gas, liquid or solid) should rapidly develop net negative charge asymmetry due to electron scattering processes and positron annihilation. The net electronic charge excess was estimated to be $\sim 20-30 \%$, and Askaryan proposed that Cherenkov radiation at wavelengths larger than the longitudinal dimensions of the shower $(\sim 1 \mathrm{~m}$ in air, and $\sim 1 \mathrm{~cm}$ in liquids or solids) would be emitted coherently, yielding a quadratic scaling of received power with the shower energy. This latter property immediately suggests that radio emission might dominate the secondary radiation at the highest energies. We defer discussion of this so-called Askaryan effect in solids to a later section; however, its application to air showers was immediately noticed and pursued. 


\subsubsection{History}

Radio emission from cosmic ray air showers was discovered for the first time by Jelley and coworkers in 1965 at a frequency of $44 \mathrm{MHz}$. They used an array of dipole antennas in coincidence with Geiger counters. The results were soon verified and emission from $2 \mathrm{MHz}$ up to $520 \mathrm{MHz}$ was found in a flurry of activities in the late 1960's. These activities ceased almost completely in the subsequent years due to several reasons: difficulty with radio interference, uncertainty about the interpretation of experimental results, and the success of other techniques for air shower measurements.

The radio properties of air showers are summarized in an excellent and extensive review by Allan (1971). The main result of this review can be summarized by an approximate formula relating the received voltage of air showers to various parameters, where we also include the presumed frequency scaling:

$$
\begin{aligned}
\epsilon_{\nu}= & 20 \mu \mathrm{V} \mathrm{m}^{-1} \mathrm{MHz}^{-1}\left(\frac{E_{\mathrm{p}}}{10^{17} \mathrm{eV}}\right) \sin \alpha \times \\
& \cos \theta \exp \left(\frac{-R}{R_{0}(\nu, \theta)}\right)\left(\frac{\nu}{55 \mathrm{MHz}}\right)^{-1} \cdot(1)
\end{aligned}
$$

Here $E_{\mathrm{p}}$ is the primary particle energy, $R$ is the offset from the shower center and $R_{0}$ is around $110 \mathrm{~m}$ at $55 \mathrm{MHz}, \theta$ is the zenith angle, $\alpha$ is the angle of the shower axis with respect to the geomagnetic field, and $\nu$ is the observing frequency (see also Allan et al. 1970; Hough \& Prescott 1970). The leading factor of 20 has been disputed over the years since it was first published, and could be an order of magnitude smaller. ${ }^{1}$

The voltage of the unresolved pulse in the coherent regime $(\nu \leq 100 \mathrm{MHz})$ can be converted into an equivalent flux density (a flux density for a steady continuum source required to produce the same energy over the bandwidth limited time interval $\Delta t$ ) in commonly used radio astronomical units

\footnotetext{
${ }^{1}$ More likely, the controversy over this coefficient probably stems from the wide variation in measurement conditions and the uncertainties in the flux calibration of the radio antennas as well as in the energy calibration of the particles.
}

$$
\begin{aligned}
S_{\nu}= & \epsilon_{\nu}^{2} \epsilon_{0} c / \Delta t \\
S_{\nu}= & 27 \mathrm{MJy} \times \\
& \left(\frac{\epsilon_{\nu}}{10 \mu \mathrm{V} \mathrm{m}^{-1} \mathrm{MHz}^{-1}}\right)^{2}\left(\frac{\Delta t}{\mu s}\right)^{-1} .
\end{aligned}
$$

The pulse duration is $\Delta t \sim 1 / \Delta \nu$ if the measurement is bandwidth-limited. Note, that for larger bandwidths and hence higher time resolution the energy of the pulse itself does not increase, however, the equivalent flux density of a steady source needs to increase, in order to produce an energy comparable to the pulse in the shorter time interval. In the earlier measurements the pulses were always unresolved when observing with $\Delta \nu \simeq 1 \mathrm{MHz}$.

The formula was determined experimentally from data in the energy regime $10^{16} \mathrm{eV}<$ $E_{\mathrm{p}}<10^{18} \mathrm{eV}$. The flux density around $100 \mathrm{MHz}$ seems to depend on primary particle energy as $S_{\nu} \propto E_{\mathrm{p}}^{2}$ (Hough \& Prescott 1970; Vernov et al. 1968; Fig. 6) as expected for coherent emission (see below). This dependency is, however, not yet undoubtedly established, since a few earlier measurements apparently found somewhat flatter power-laws (Barker et al. 1967 as quoted in Allan 1971).

Very little concrete data exist on the spectral dependence of EAS (Extensive Air Shower) radio emission (e.g., Spencer 1969). Figure 7 shows a tentative EAS radio spectrum with a $\nu^{-2}$ dependence for the flux density $\left(\nu^{-1}\right.$ dependence for the voltage). The $2 \mathrm{MHz}$ data point was made with a different experiment and there is a possibility that the spectrum is somewhat flatter between 10-100 $\mathrm{MHz}$ (see Datta et al. 2000), but this is not verified. The polarisation of the emission could be fairly high and is basically along the geomagnetic E-W direction (Allan, Neat, \& Jones 1967) which strongly supports an emission mechanism related to the geomagnetic field.

Finally, one needs to consider the spatial structure of the radio pulse. The current data strongly supports the idea that the emission is not isotropic but is highly beamed in the shower 


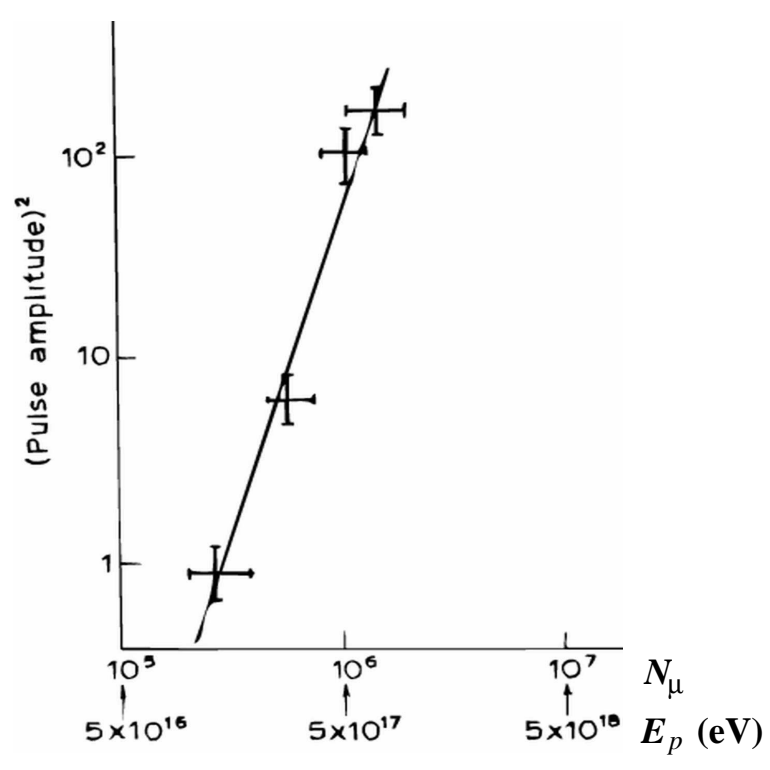

Figure 6. The dependence of EAS radio flux on the primary particle energy as measured by Vernov et al. (1968) following roughly a $E_{\mathrm{p}}^{2}$ powerlaw. Some earlier papers found somewhat flatter dependencies.

direction. Figure 8 shows EAS radio pulse amplitude measurements as a function of distance $R$ from the shower axis - the flux density drops quickly with offset from the center of the shower. The characteristic radius of the beam is of order 100 meter for a $10^{17} \mathrm{eV}$ vertical shower, with the emission originating at 5-7 km distance above an observer at sea level. The implied angular diameter of the beam is thus $\Theta \simeq 0.2 / 6=1.9^{\circ}$.

\subsubsection{The synchrotron model and recent work.}

Experiments have clearly established that cosmic ray air showers produce radio pulses. The original motivation was due to a suggestion from Askaryan (1962) who argued that annihilation of positrons would lead to a negative charge excess in the shower, thus producing Cherenkov radiation as it rushes through the atmosphere. At radio frequencies the wavelength of the emission

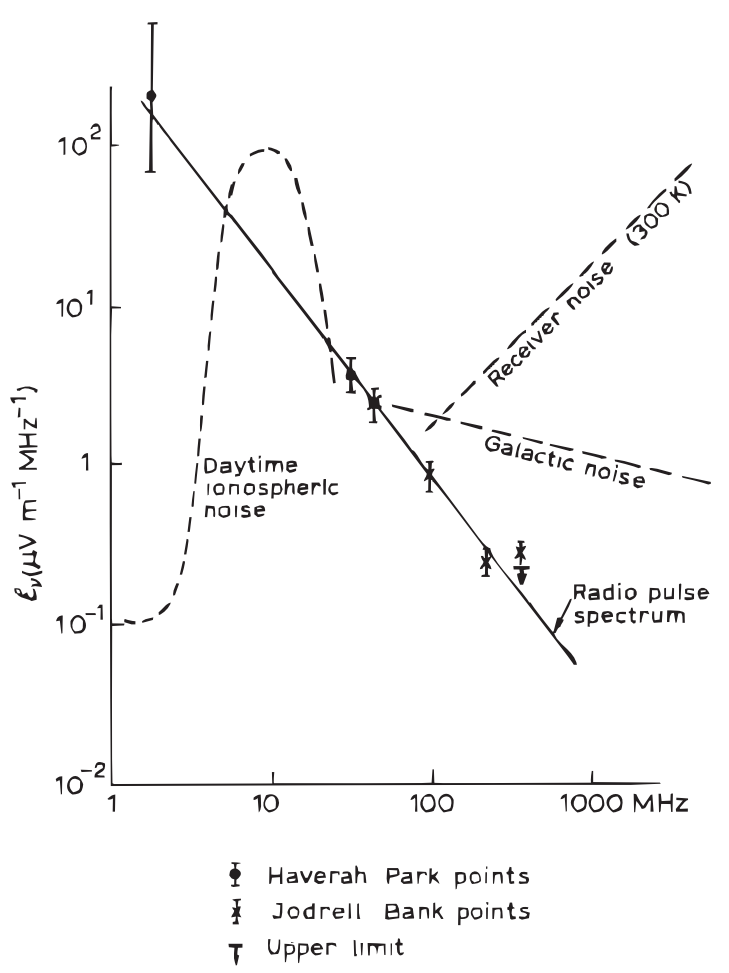

Figure 7. A tentative radio pulse spectrum for 2 MHz to $520 \mathrm{MHz}$. The data are not simultaneous. From Allan (1971) and Spencer (1969).

is larger than the size of the emitting region and the emission should be coherent. The radio flux would then grow quadratically with the number of particles rather than linearly and thus would be greatly enhanced. This effect is important in dense media where it was already experimentally verified (Saltzberg et al. 2001; see below) and is important for detecting radio emission from neutrino showers in ice or on the moon.

However, the dependence of the emission on the geomagnetic field detected in several later experiments indicates that another process may be important. The basic view in the late 60 's was that the continuously created electron-positron pairs were then separated by the Lorentz force in the geomagnetic field which led to a transverse current in the shower. If one considers a 


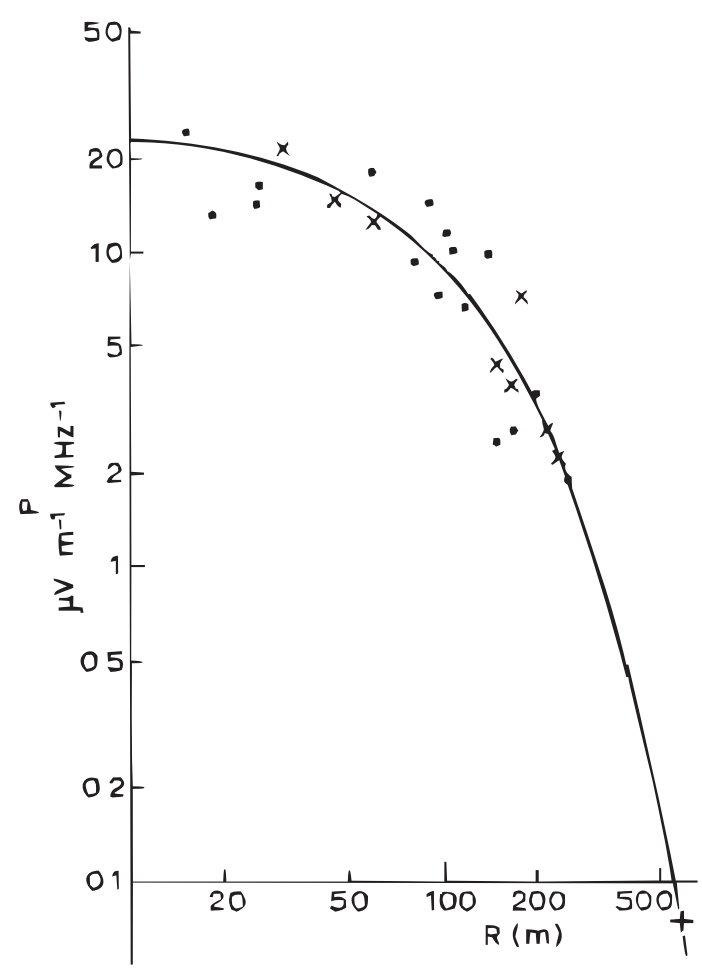

Figure 8. Normalized radio pulse amplitudes in $\mu \mathrm{V} \mathrm{m}^{-1} \mathrm{MHz}^{-1}$ at $55 \mathrm{MHz}$ as a function of distance $R$ in meters from the shower axis. Each data point corresponds to one measured cosmic ray event. The amplitudes were normalized to a reference energy of $E_{\mathrm{p}}=10^{17} \mathrm{eV}$ assuming the above mentioned linear dependence of voltage on primary particle energy. The measurements were made for zenith angles $\theta<30^{\circ}$. Crosses and dots represent different particle energy bins between $10^{17} \mathrm{eV}$ and $10^{18} \mathrm{eV}$. The plus sign at 500 meters marks a single $10^{19} \mathrm{eV}$ event. From Allan (1971).

frame moving along with the shower, one would observe electrons and positrons drifting in opposite directions impelled by the transverse electric field induced by the changing geomagnetic flux swept out by the shower front. (Only in the case of shower velocity aligned with the magnetic field lines will this induced electric field vanish). This transverse current then produces dipole (or Lar- mor) radiation in the frame of the shower. When such radiation is Lorentz-transformed to the lab frame, the boost then produces strongly forwardbeamed radiation, compressed in time into an electro-magnetic pulse (EMP). This was calculated by Kahn \& Lerche (1966) and also Colgate (1967).

Falcke \& Gorham (2003) suggested it might be better to think of the emission simply as being synchrotron-like in the earth's magnetic field, or "coherent geosynchrotron emission", as they called it. This process is probably equivalent to the previous suggestions since it is derived from the basic formula for dipole radiation and the Poynting vector but does not require a consideration of charge separation: The different sign of the charges is canceled by the opposite sign in the Lorentz force for electrons and pairs and hence both contribute in exactly the same way to the total flux (radio astronomers will surely remember that an electron/positron plasma produces almost the same amount of synchrotron emission as a pure electron plus proton plasma).

The basic and intuitive derivation of this effect can be found in Falcke \& Gorham (2003) using standard synchrotron radiation theory. One important effect which is explicitly neglected by this simple treatment is the Fresnel zone problem - vertical air showers at $10^{19} \mathrm{eV}$ reach their particle maximum at ground level, and the radio emission arrive nearly simultaneously to the particle "pancake," indicating that the far-field conditions, where the radiation field has had time to become well-separated from its source, are not satisfied. Any estimate of the details of the received radio emission which is intended to help with detailed detector design, such as what may be required to justify any impact on SKA parameters or planning, must therefore treat the problem with much greater fidelity.

Such high-fidelity simulations of geosynchrotron emission are now beginning to appear in the literature, and as the interest in this approach grows, along with the compelling nature of the ultra-high energy cosmic ray problem, the simulations can be expected to improve as well. In the following section, we describe recent results in this direction. 


\subsubsection{Air shower electrodynamics: de- tailed modeling and Monte Carlo simulation.}

The challenge of developing high-fidelity air shower radio simulations breaks into three distinct problems:

1. The adaptation of existing air shower simulation codes to provide the particle identification and sampling needed for electrodynamics modeling;

2. The implementation of actual electrodynamics computation within the modified air shower code, and the development of radiation propagation model; and

3. the modeling of the detector geometry and detection process.

To date, no group has implemented all three aspects of this program, but we describe here two efforts which have gone much further than others in addressing the difficult problem of the electrodynamics and detection modeling.

\section{Simulations by the Chicago/Hawaii group.}

One result with a first-order electrodynamics Monte Carlo simulation has been completed by Suprun et al. (2003) in a joint effort of the Univ. of Chicago group headed by Jon Rosner, along with one of the current authors (P. Gorham) of this chapter. This study investigated a $10^{19} \mathrm{eV}$ vertical air shower, including explicit geomagnetic effects, with general interest in elucidating issues for detection by a possible radio augmentation to the Auger Observatory for ultra-high energy cosmic rays.

The Suprun et al. simulation did not make any simplifying assumptions regarding far-field conditions. Instead, the electrodynamics simulation began with the general formula for a radiating particle (Jackson 1999, Zas et al. 1992) in arbitrary motion:

$\mathbf{E}\left(\mathbf{x}, t_{a}\right)=\frac{e \mu}{4 \pi \epsilon_{0}}\left[\frac{\mathbf{n}-n \boldsymbol{\beta}}{\gamma^{2}|1-n \boldsymbol{\beta} \cdot \mathbf{n}|^{3} l^{2}}\right]_{\mathrm{ret}}+$

$$
\frac{e \mu}{4 \pi \epsilon_{0} c}\left[\frac{\mathbf{n} \times[(\mathbf{n}-n \boldsymbol{\beta}) \times \dot{\boldsymbol{\beta}}]}{|1-n \boldsymbol{\beta} \cdot \mathbf{n}|^{3} l}\right]_{\mathrm{ret}}
$$

which is correct regardless of the distance to the antenna. In this formula $\boldsymbol{\beta}$ is the velocity vector in units of $c, \dot{\boldsymbol{\beta}}=d \boldsymbol{\beta} / d t$ is the acceleration vector, divided by $c, \mathbf{n}$ is a unit vector from the radiating particle to the antenna, and $l$ is the distance to the particle. $\mu \approx 1$ denotes the relative magnetic permeability of air, $n$ the index of refraction. The square brackets with subscript "ret" indicate that the quantities in the brackets are evaluated at the retarded time, not at the time $t_{a}$ when the signal arrives at the antenna.

The first term decreases with distance as $1 / l^{2}$ and represents a boosted Coulomb field. It does not produce any radiation. The magnitudes of the two terms in Eq. (4) are related as $1 /\left(\gamma^{2} l\right)$ and $|\dot{\boldsymbol{\beta}}| / c$. The characteristic acceleration of a $30 \mathrm{MeV}$ electron $(\gamma \approx 60)$ of an air shower in the Earth's magnetic field $(B \approx 0.5$ Gauss $)$ is $|\mathbf{a}|=e c B /(\gamma m) \approx 4.4 \cdot 10^{13} \mathrm{~m} / \mathrm{s}^{2}$. Even when an electron is as close to the antenna as $100 \mathrm{~m}$, the first term is two orders of magnitude smaller than the second and can be neglected. The second term falls as $1 / l$ and is associated with a radiation field. It describes the electric field of a single radiating particle for most geometries relevant to extensive air showers. It can be shown (Wheeler \& Feynmann 1949) to be proportional to the apparent angular acceleration of the charge up to some non-radiative terms that are proportional to $1 / l^{2}$. This relation is referred to in the literature as "Feynman's formula."

Suprun et al. did not, however, yet perform a full cascade calculation, but rather used a parametrisation of the shower density to generate a shower profile, then used Monte Carlo techniques to sample the particle distribution obeying this parametrisation. In one of the longeststanding empirical models for air shower development, called the Nishima, Kamata, Greisen (NKG) model, the lateral particle density $\rho_{e}$ is parametrized by the age parameter $s$ of the shower ( $s=1$ for the shower maximum) and the Molière radius $r_{m}$ (Bourdeau et al. 1980, Greisen 
1956, Kamata \& Nishimura 1958):

$\rho_{e}=K_{N}\left(\frac{r}{s_{m} r_{m}}\right)^{s-2}\left(1+\frac{r}{s_{m} r_{m}}\right)^{s-4.5}$,

where

$K_{N}=\frac{N}{2 \pi s_{m}^{2} r_{m}^{2}} \frac{\Gamma(4.5-s)}{\Gamma(s) \Gamma(4.5-2 s)}$,

$\Gamma$ is the gamma function, $r$ the distance from the shower axis, $N$ the total number of charged particles, and $s_{m}=0.78-0.21 s$. The Molière radius for air is approximately given by $r_{m}=$ $74\left(\rho_{0} / \rho\right) \mathrm{m}$, with $\rho_{0}$ and $\rho$ being the air densities at sea level and the altitude under consideration, respectively.

As a shower travels toward the Earth and enters denser layers of the atmosphere, the age parameter increases while the Molière radius drops. Both processes affect the spread of the lateral distribution. The influence of the age parameter appears to be more significant. As it grows, the average distance of the shower particles from its axis increases. This effect overcomes the influence of a smaller Molière radius which tends to make the lateral distribution more concentrated toward the axis. For a fixed age parameter $s$, however, the Molière radius is the only quantity that determines the spread of the lateral distribution. At shower maximum $(s=1)$ the average distance from the axis can be calculated to be $(2 / 3) s_{m} r_{m}=0.38 r_{m}$.

Fig. 9 shows the results of the Suprun et al. simulation for the shape of the intrinsic radio pulse, in terms of field strength vs. time at the receiving antenna location, though without any of the filtering effects of any antenna imposed on it yet. Fig. 10] gives the Fourier transform $E_{\nu}$ of this pulse. The nonzero thickness of the airshower pancake translates into a loss of coherence at frequencies corresponding to wavelengths comparable to the shower thickness, thereby limiting the main part of the radiation spectrum to the frequencies below $100 \mathrm{MHz}$.

These simulations, though using a greatly thinned set of input particles $\left(10^{4}\right.$ compared to $10^{10}$ in actuality) do show characteristics similar to what was observed historically. In addition, the simulations also begin to reveal some of the

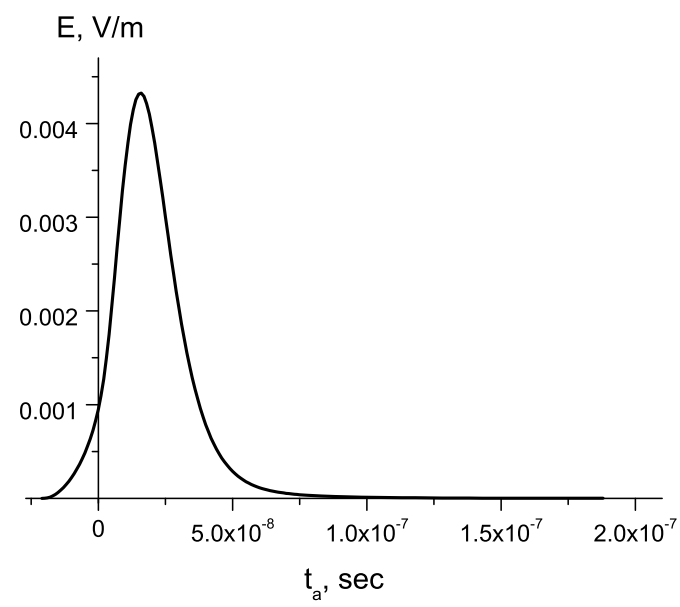

Figure 9. The EW component $E_{E W}$ of electromagnetic pulse of $0.33 \cdot 10^{10}$ radiating electronpositron pairs distributed over the thickness of the shower pancake at $1800 \mathrm{~m}$ above sea level. The axis of the pancake is located $200 \mathrm{~m}$ South of the antenna. The time axis was chosen in such a way that the pulse produced by a pair located in the axis at the bottom of the pancake starts at time 0 .

geomagnetic complexity of the emission pattern, suggesting reasons for some of the surprising variations observed in the measurements by antenna arrays.

Consider the frame centered at the antenna, with axis $O x$ going to the magnetic West, $O y$ to the South and $O z$ directly up. The initial velocity of all charged particles is assumed to be vertical: $\boldsymbol{\beta}=(0,0,-1)$, while the initial acceleration $\dot{\boldsymbol{\beta}}$ is parallel to $O x$, or, in other words, to the $(1,0,0)$ vector.

Electrons bend toward the magnetic West and positrons toward the East. The electric fields from both particles of an electron-positron pair are coherent; the opposite signs of their accelerations are canceled by the opposite signs of the electric charges. 


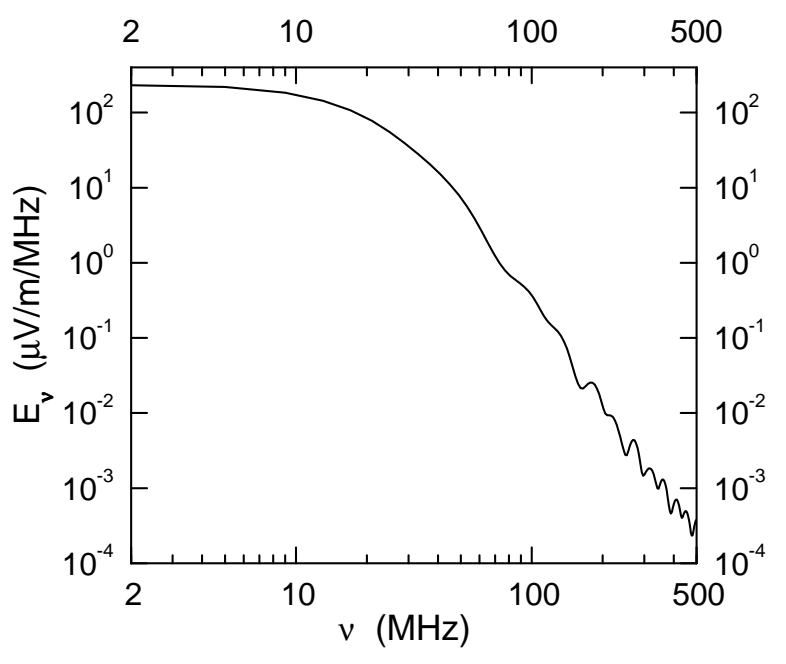

Figure 10. The Fourier transform of the electromagnetic pulse shown in Fig. 9 The spectrum is very flat below $2 \mathrm{MHz}$. The limited statistics of the model results in some jitters at 200-500 MHz. The spectrum above $500 \mathrm{MHz}$ is not shown because the statistics is not sufficient to make reliable predictions of the Fourier components at these high frequencies.

Let $\psi$ be the angle between $O x$ and the direction to the shower core, $R$ the distance to the core, and $h$ the altitude of the radiating particle above the antenna. The denominator of the second term of Eq. (4) is independent of $\psi$. The numerator determines that, to leading (second) order in $R / h$, the initial electric field vector $\mathbf{E}$ received at the antenna lies in the horizontal plane and is parallel to $(\cos 2 \psi, \sin 2 \psi, 0)$ (Green et al. 2003):

$\mathbf{E} \|(\cos 2 \psi, \sin 2 \psi, 0)$.

The magnitude of the numerator is independent of the angle $\psi$ up to terms of order $R^{4} / h^{4}$. This result shows that although particles are accelerated by the Earth's magnetic field in the EW direction regardless of angle $\psi$, the radiation received at the antenna does not show preference for the EW polarisation. Instead, it is directly related to the angle $\psi$. As the particle trajectory bends in the Earth's magnetic field and the velocity deflects from the vertical direction, the relation (77) between the direction of the electric field vector and angle $\psi$ does not hold. Nonetheless, it will be useful for understanding the angular dependence of the electric field.

Suprun et al. computed electromagnetic pulses for the pancakes with axes located at the same distance $R=200 \mathrm{~m}$ from the antenna but at various angles $\psi$ from the $O x$ direction. Fig. 111 shows the radio signal strengths that would be received by EW and NS-oriented antennas. Note that Eq. (7) predicts that components of the radiation coming from the start of the particle trajectory vanish at some angles $\psi: E_{E W}=0$ at $\psi=$ $\pm \pi / 4, \pm 3 \pi / 4$, while $E_{N S}=0$ at $\psi=0, \pm \pi / 2, \pi$. This fact explains why $E_{\nu E W}$ is relatively small at $\psi= \pm \pi / 4, \pm 3 \pi / 4$ and $E_{\nu N S}$ is small at $\psi=0, \pi$ (Fig. 111). Another mechanism is responsible for $E_{\nu N S}$ being virtually 0 at $\psi= \pm \pi / 2$. At these angles the trajectories of two charged particles of an electron-positron pair are symmetric with respect to the $y O z$ plane. The NS component of radiation emitted by this pair vanishes not only at the start but throughout its flight.

\section{Modeling by the LOPES collaboration.}

Another simulation effort is under way in the Max-Planck-Institut fr Radioastronomie at Bonn, led by T. Huege and an author of this chapter (H. Falcke). This group is part of a collaboration developing the LOFAR Prototype Experimental Station (LOPES), an engineering model of one station of the Low Frequency Array (LOFAR). LOPES is operating jointly with the KASCADE Grande air shower array in Karlsruhe (Schieler et al. 2003); LOFAR is a funded effort to develop a very large area ground array for radio astronomy in the HF to VHF regime, sharing many common interests with the SKA. The LOPES group has recently published a detailed analysis of the geosynchrotron model for the case of a $10^{17} \mathrm{eV}$ air shower (Huege \& Falcke 2002, 2003) in preparation for a major effort at an electro-dynamical air shower Monte Carlo code (Huege \& Falcke 2004).

The LOPES group has taken special care of taking into account the longitudinal development of the air shower by performing an integration 


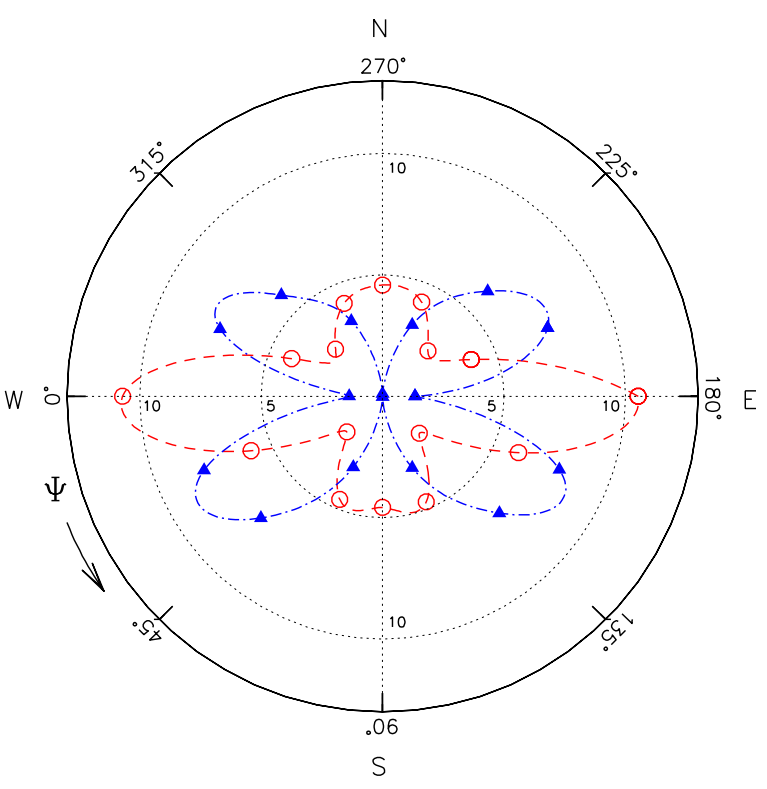

Figure 11. The East-West and North-South components of the field strength $\left|E_{\nu E W}\right|$ and $\left|E_{\nu N S}\right|$ (circles and triangles, respectively) at $55 \mathrm{MHz}$ as functions of angle $\psi$ between the magnetic West and direction to the shower core. The distance between the origin and a circle or a triangle represents the field strength in the units of $\mu \mathrm{V} / \mathrm{m} / \mathrm{MHz}$. The angular spacing between circles or triangles is $\pi / 8$. At $\psi= \pm \pi / 2\left|E_{\nu N S}\right|$ do not exceed $0.1 \mu \mathrm{V} / \mathrm{m} / \mathrm{MHz}$ and two triangles overlap. All points were calculated for the vertical shower at a $200 \mathrm{~m}$ distance from the antenna.

over the shower as a whole, and they have considered the variation of the field strength as a function of radial distance from the shower core as well. They use a shower parametrisation based on the NKG model with a shower disk that flares out from the center, in a manner similar to the Chicago/Hawaii study, and thus, apart from the energy difference, the results do bear some comparison. The LOPES study also did an integral over a power-law distribution of electron energies, appropriate to an air shower. However, they did not do any near-field corrections to their results, but this is not a major drawback for a lower energy shower since these showers do reach their maxima at altitudes of typically several $\mathrm{km}$ away from an observer on the ground.

Fig. 12 shows the spectrum emitted by the air shower maximum for a shower disk profile with realistic flaring according to the parametrisations of Agnetta et al. (1997) and Linsley (1986). As expected, the spectrum emitted by the Linsley flaring disk extends to higher frequencies than the one generated by the Agnetta flaring disk because of the lower thickness in the shower centre where most of the particles reside.

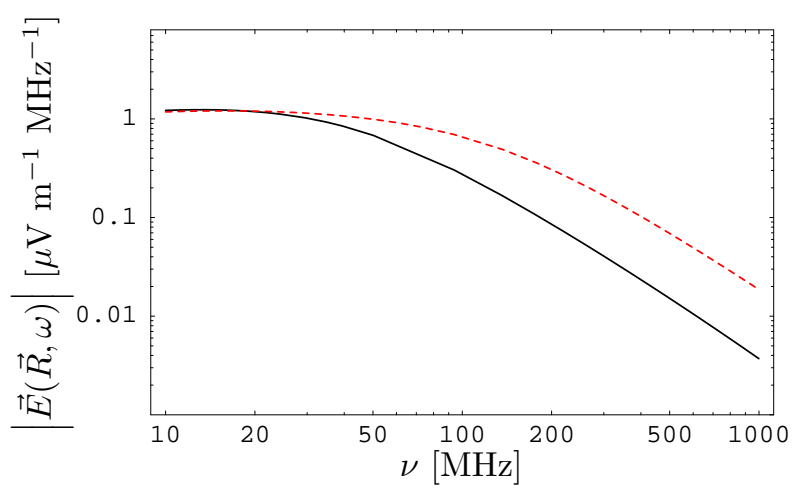

Figure 12. $|\vec{E}(\vec{R}, 2 \pi \nu)|$-spectrum at the centre of the area illuminated by the maximum of a $10^{17} \mathrm{eV}$ air shower with flaring $\Gamma$-pdf, $R_{0}=4 \mathrm{~km}$ and a broken power-law energy distribution from $\gamma=5-1000$. Solid: flaring (Agnetta et al. 1997) lateral distribution, short-dashed: flaring (Linsley 1986) lateral distribution

The modeled radial dependence at different frequencies is shown in Figure 13 Here the three families of curves represent different frequencies, and the different slopes between the two curves at a given frequency are for the cases of an observer with a given distance from the shower center in the directions perpendicular and parallel to the geomagnetic field. This result thus indicates again the importance of the geomagnetic 
effects in the azimuthal distribution of radiation for a given magnetic field direction. Early results from the upcoming detailed Monte Carlo simulations of the LOPES collaboration, however, show that asymmetries in the emission pattern due to the geomagnetic field seem to be washed out to a high degree once realistic distributions of particle track lengths are taken into account.

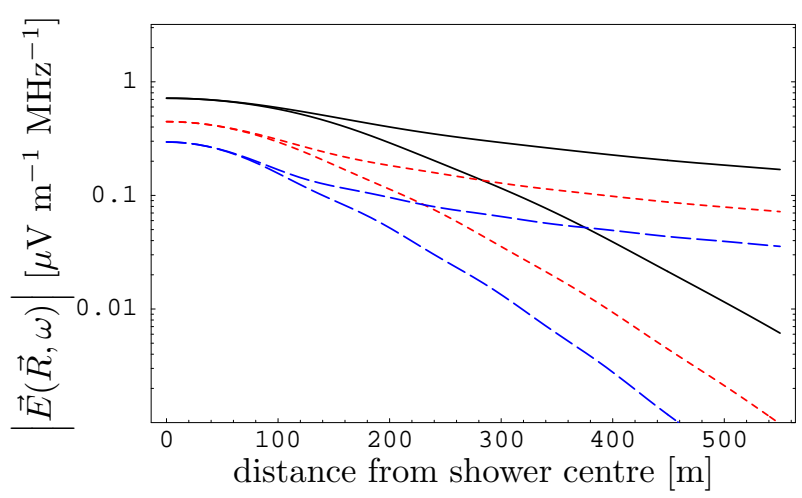

Figure 13. Radial dependence of $|\vec{E}(\vec{R}, 2 \pi \nu)|$ for the maximum of a $10^{17} \mathrm{eV}$ air shower with flaring (Agnetta et al. 1997) $\Gamma$-pdf, $R_{0}=4 \mathrm{~km}$ and a broken power-law energy distribution from $\gamma=$ 5-1000. Solid: $\nu=50 \mathrm{Mhz}$, short-dashed: $\nu=$ $75 \mathrm{Mhz}$, long-dashed: $\nu=100 \mathrm{Mhz}$, upper curves for distance from shower center to the east-west, lower curves for distance to north-south.

Fig. 14] shows a reconstructed pulse generated by the flaring Agnetta disk as it would be measured by a receiver with a given bandwidth. The pulse amplitude drops noticeably when the observer moves from the centre of the illuminated area on the ground to a distance of $100 \mathrm{~m}$, and is already quite diminished at a distance of $250 \mathrm{~m}$.

The LOPES study addresses the important problem of integrating over the shower evolution as a whole in a simplified fashion by approximating the shower evolution with a number of discrete steps. The characteristic scale

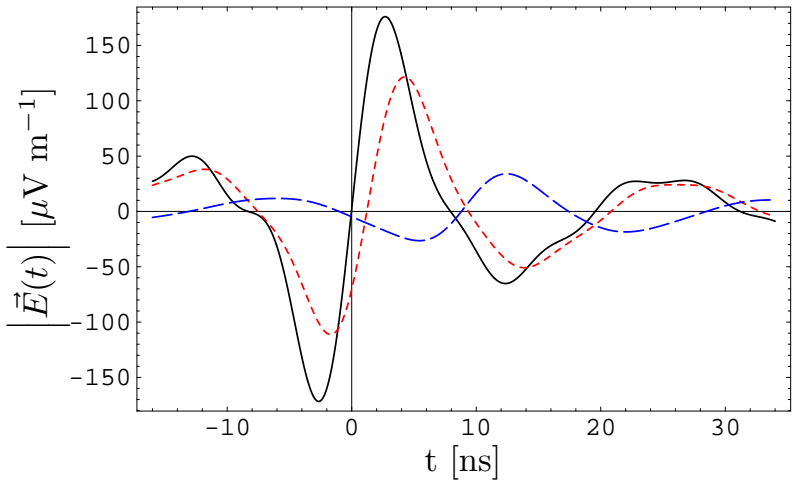

Figure 14. Reconstructed pulses emitted by the maximum of a $10^{17} \mathrm{eV}$ shower with flaring (Agnetta et al. 1997) $\Gamma$-pdf, broken power-law energy distribution from $\gamma=5-1000$ and $R_{0}=4 \mathrm{~km}$, using an idealized rectangle filter spanning 40 $160 \mathrm{MHz}$. Solid: centre of illuminated area, shortdashed: $100 \mathrm{~m}$ to north from centre, dash-dotted: $250 \mathrm{~m}$ to north from centre

for these steps is given by the "radiation length" of the electromagnetic cascades in air, $X_{0}=$ $36.7 \mathrm{~g} \mathrm{~cm}^{-2}$, corresponding to $\approx 450 \mathrm{~m}$ at a height of $4 \mathrm{~km}$. One can therefore discretise the shower evolution into "slices" of thickness $X_{0}$, assuming these contain independent generations of particles and therefore radiate independently. Superposition of the individual slice emissions, correctly taking into account the phases arising from arrival time differences, then leads to the total emission of the shower.

For a vertical $10^{17} \mathrm{eV}$ air shower at a height of $R_{0}=4 \mathrm{~km}$ they add the emission from eight slices above and eight slices below the shower maximum to the emission from the maximum itself. The closest slice then lies at $R_{0}=950 \mathrm{~m}$ from the observer, a distance they did not want to fall below because of approximations contained in their calculations that are only valid in the far-field.

Although this treatment is clearly oversimplified, the results depicted in Fig. [15] indicate that the integration over the shower as a whole significantly enhances the emission strength and thus 
cannot be neglected. In particular, this implies that the emission is actually not dominated by a narrow region around the shower maximum, but that the entire shower evolution contributes. A realistic treatment of the integration over the shower as a whole is carried out as part of the upcoming Monte Carlo simulations of the LOPES collaboration (Huege \& Falcke 2004).

Data from the LOPES Experiment will become available soon, but first results indeed confirm the association of the air shower with a sharp radio pulse, having the expected properties (e.g., Horneffer et al. 2004). This puts the radio detection method on rather firm ground.

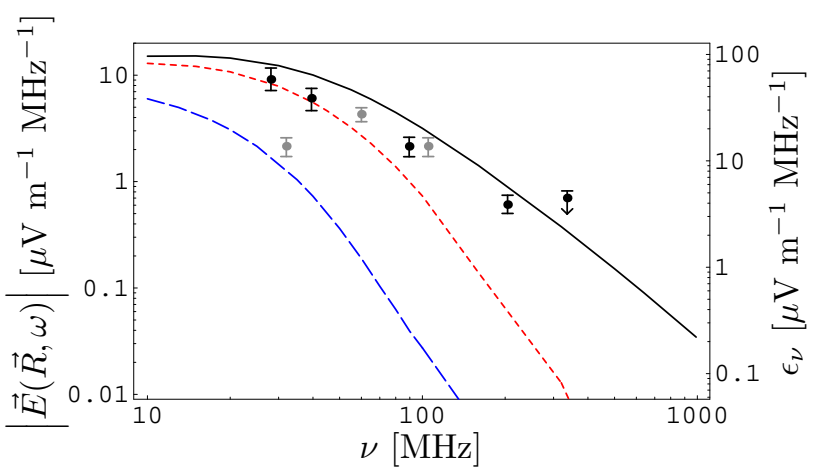

Figure 15. $\quad|\vec{E}(\vec{R}, 2 \pi \nu)|$-spectrum of a full (longitudinally integrated) $10^{17} \mathrm{eV}$ air shower with flaring (Agnetta et al. 1997) $\Gamma$-pdf, $R_{0}=4 \mathrm{~km}$ and a broken power-law energy distribution from $\gamma=5-1000$. Solid: centre of illuminated area, short-dashed: $100 \mathrm{~m}$ to north from centre, longdashed: $250 \mathrm{~m}$ to north from centre, black points: re-scaled (Spencer 1969) data as presented by (Allan 1971), grey points: re-scaled (Prah 1971) data

It is interesting that in spite of the differences in the approach from the LOPES studies and those of the Chicago/Hawaii group, the results for the radio spectrum for a distance of $200 / 250 \mathrm{~m}$ from the shower core show a very similar frequency de- pendence, with the field strength falling about a factor of 300 as one goes from 10 to $100 \mathrm{MHz}$. The absolute value of the field strength is about a factor of 30 or so different, which is inconsistent with a strict linear scaling of field strength with energy as one might expect; however, agreement to within a factor of 2-3 is actually quite good considering the fact that these are completely independent efforts.

\subsection{Askaryan effect and its confirmation}

As noted early in this discussion, the Askaryan effect was the original motivation for much of the effort to measure radio emission from air showers, but the coherent geo-synchrotron emission detailed above was found to be the dominant contribution for air showers, and the coherent Cherenkov emission from the charge excess, while not discounted, was largely forgotten because of its small contribution. However, for showers in solid materials such as ice or the lunar regolith which are relatively radio-transparent, the shower lengths are short enough $(\sim 10 \mathrm{~m})$ that the magnetic effects leading to synchrotron emission may be neglected, and the coherent Cherenkov emission becomes the more important secondary radiation. Here the quadratic rise of radio power with frequency leads to the conclusion that, at energies above $10^{18} \mathrm{eV}$, the coherent Cherenkov emission will dominate all secondary radiation, including optical emission, by a wide margin.

Although it is not presently possible to produce $\mathrm{EeV}$ cascades in terrestrial accelerators, electromagnetic showers with composite total energies in this range can be easily synthesized by superposing gamma-rays of energies above the pairproduction threshold. If the gamma-ray bunch is small compared to the wavelength of the radio emission (true for most pulsed linacs), the resulting showers will differ from natural $\mathrm{EeV}$ showers only logarithmically, due to the details of the initial interaction. However, since the bulk of the radio emission arises from the region of maximum shower development, the differences in radio Cherenkov emission are modest and easily quantified.

In mid-2000, Askaryan's hypothesis was in fact confirmed at the Stanford Linear Accelerator 
Center (SLAC) in an experiment using a silicasand target and pulsed gamma-ray bunches with composite energies in the $\mathrm{EeV}$ range (Saltzberg et al. 2001). In the 2002 follow-on experiment (Gorham et al. 2004), the sand was replaced by synthetic rock salt, which has a higher dielectric constant and lower loss tangent than silica sand, and further studies were made of the polarisation behavior of the emission.

Fig. 16A shows a typical pulse profile (inset) and a set of measured peak field strengths for pulses taken at different points along the shower in the 2000 experiment. The plotted curve shows the expected profile of the total number of particles in the shower, based on the Kamata-Nishimura-Greisen approximation (Saltzberg 2001). Here the field strengths have been scaled in the plot to provide an approximate overlay to the relative shower profile. Clearly the pulse strengths are highly correlated to the particle number profile. Since the excess charge is also expected to closely follow the shower profile, this result confirms Askaryan's hypothesis.

Pulse polarisation was measured with an Sband $(2 \mathrm{GHz})$ horn directed at a shower position $0.5 \mathrm{~m}$ past the shower maximum. Fig. $16 \mathrm{~B}$ shows the pulse profile for both the $0^{\circ}$ and $90^{\circ}$ (cross-polarised) orientations of the horn. The lower two panes of this portion show the derived degree of linear polarisation and the angle of the plane of polarisation, respectively. Because of the vector correlation of the pulse polarisation with the shower velocity vector and the Poynting flux vector, it is possible to use the angle of the polarisation to track the shower axis. An example of this is shown in Fig. 16] C, where the angle of the plane of polarisation is plotted at three locations with respect to the shower axis, showing the high correlation with the predicted angle.

Fig. 16D shows a typical sequence of pulse field strengths versus the total shower energy. The fitted linear rise of field strength with beam current is consistent with complete coherence of the radiation, implying the characteristic quadratic rise in the corresponding pulse power with shower energy. Fig. 16F shows a similar result for the 2002 experiment, but now covering a much wider range of energy, plotted as pulse power instead of field strength. The Askaryan process is found to be quadratic over four orders of magnitude in shower energy.

Fig. 16 $\mathrm{E}$ shows the spectral dependence of the radiation, which is consistent with the linear rise with frequency that is also characteristic of Cherenkov radiation. Also shown is a curve based on a parametrisation of Monte Carlo results (Zas et al. 1992). The uncertainties are estimates of the combined systematic and statistical uncertainties. Note that the figure compares absolute field strength measurements to the predictions and the agreement is very good.

In summary, there is clear experimental evidence that Askaryan's hypothesis is confirmed and that the predicted emission from high energy cascades is present in the expected amounts. This lends strong support to experiments designed to exploit this effect for high energy neutrino and cosmic ray detection.

\section{Prospects for the SKA}

The ultra-fast transient radio events described here will either be signal, or at some level, background for the SKA. With recent pulsar studies extending to broader and broader bandwidths, and faster and faster pulse transients, understanding of these events may become important in verifying the detection of pulsar transient events, certainly a mainstay of SKA scientific interest. Whether or not the SKA can be a competitive instrument for the detection of the various types of events described here will depend strongly on the final choice of design. However, without careful choices made at this early stage of the effort, it will be much more likely that the design is "pessimized" rather than optimized for their detection.

\subsection{Cosmic ray air shower detection}

From the description above, it is evident that air shower radio emission in the $\geq 10^{18} \mathrm{eV}$ energy regime has three important characteristics which will impact the design of any radio array with intent to detect them:

- The optimal frequency range is $\sim 20-$ 

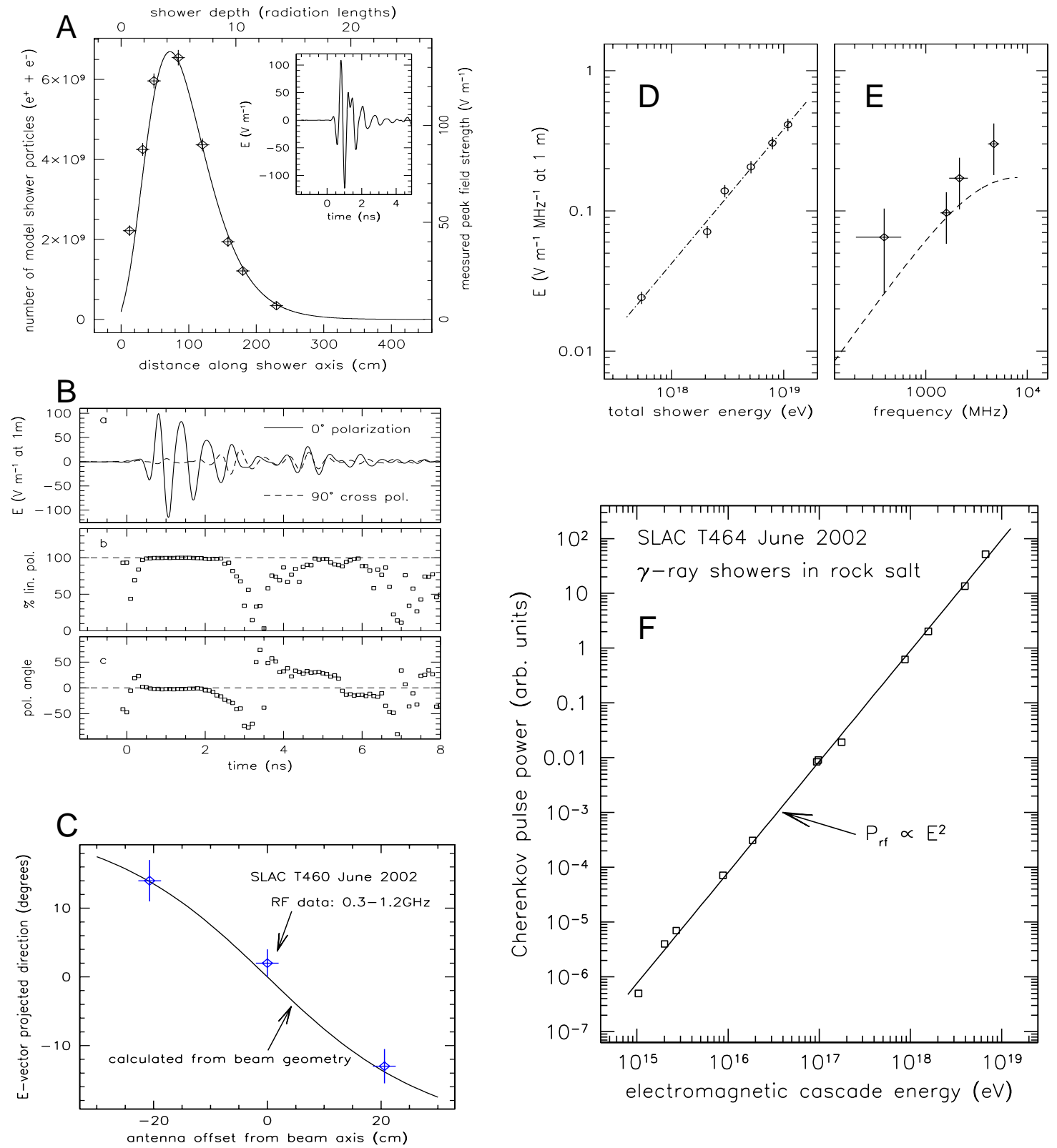

Figure 16. A: Shower RF field strength profile with typical pulse (inset). B: Polarisation measurements of a typical RF Cherenkov pulse at $2 \mathrm{GHz}$. C: Correlation of plane of polarisation with antenna offset from shower axis. D: Coherence of RF Cherenkov at $2 \mathrm{GHz}$, measured during 2000 SLAC experiment. E: absolute field strength and prediction from Cherenkov. F: Coherence of radiated power over the 0.2-1.2 GHz band. 
$200 \mathrm{MHz}$

- The physical area over which one expects to detect the emission is limited in diameter to a few tens of $\mathrm{km}$, and often much less.

- The time-scale for the air shower radio emission is an impulse of order 20 ns or less in duration.

An SKA design extending down to $200 \mathrm{MHz}$ is still adequate, though not optimized, for air shower detection, but the sparsity of the array and the bandwidth of the front-end receivers will have significant impact on the triggering and reconstruction of the shower energy and direction, and of course the ultimate sensitivity. For the SKA to be competitive for giant air shower detection, careful consideration of all of these factors will make the difference as to whether the SKA is irrelevant for this field, or a dominant competitor.

Because of the wide variety of designs currently under consideration for the SKA, and their rapid evolution, it is impractical to assess each one for its capability in air shower radio detection. Instead, we take the approach of estimating what would make the ideal detector for air shower radio detection, and then we consider how this compares to current plans.

As noted above, one would ideally like to work at frequencies that extend below $100 \mathrm{MHz}$, but with as broad a bandwidth as possible, able even to resolve the $\sim 10 \mathrm{~ns}$ time-scale for air shower radio emission. This requires several hundred $\mathrm{MHz}$ of bandwidth, extending perhaps down to $50 \mathrm{MHz}$ or so. The immediate implication is that the fundamental detector element must be a very broad-band antenna, perhaps with of order $6: 1$ bandwidth or more. A high-gain antenna is also problematic, since one cannot predict a priori the arrival direction of an air shower radio pulse. For that reason a phased-array concept with digital beam-forming is by far superior to other designs. Dual polarisation is also clearly desirable, since the radiation itself is highly polarised.

These considerations lead one to consider scaleinvariant designs for the primary antenna element such as dual-polarisation log-periodic dipole antennas (LPDAs), but their typical beam-widths $\left(60-90^{\circ}\right)$ would require a cluster of at least 6 antennas to get even minimal coverage of the entire sky. LPDAs have one other characteristic which is undesirable for air shower detection: their inherent pulse dispersion reduces sensitivity to impulsive events unless a de-dispersing compensator either analog or digital) is implemented. However, if this can be overcome, they are lightweight, easy to construct and straightforward for impedance matching and modeling. Variations on the LPDA design could also be scaled up as stand-alone units to satisfy this need, and are more compact with potentially better phase centers. A non-dispersive alternative could be an array of quad-ridged horns, which can routinely achieve the $6: 1$ bandwidth required, but they would be larger and heavier than LPDAs. Obviously, even a simple active "inverted-V" antenna as used for LOFAR and LOPES are also very useful if optimized for the right frequencies.

The use of such a broad-band system of course raises the question of how one can possibly deal with interference. This has been successfully demonstrated with the LOPES experiment (see Horneffer et al. 2003). Another excellent example of a solution to this problem is the FORTE satellite (Lehtinen et al. 2004), which launched in 1997 with a 30-300 MHz nadir-pointing dualpolarisation LPDA with a tunable $25 \mathrm{MHz}$ receiving band. FORTE was optimized for detection of electromagnetic impulsive events, and its mission was to provide an unclassified test-bed for nuclear treaty verification efforts while pursuing a science program of lightning and atmospherics detection.

At an orbital altitude of $800 \mathrm{~km}$, FORTE was constantly exposed to a barrage of anthropogenic EM interference. FORTE was able to retain triggering capability for impulses down to a level within about $5 \sigma$ of the ambient thermal noise level by sub-dividing their large band into a series of $1 \mathrm{MHz}$ channels and triggering when a majority of the bands exceeded threshold indicating a broad-band pulse. The signal digitization was still done over the entire bandwidth, preserving the broad-band coherence of the impulse. But since the vast majority of anthropogenic interference is inherently narrow-band, the multiband trigger technique was very effective, when 
combined with a so called noise-riding threshold which effectively maintained the trigger rate for each sub-band to a constant level. This greatly reduced the ability of strong narrow-band carriers to cause rapid re-triggering of one of the channels which might skew the broad-band trigger rate. As a result, analysis of FORTE data has recently even provided the first published limits on neutrino fluxes in energy regimes of $\sim 10^{22-24} \mathrm{eV}$, based on the lack of observed radio impulses emanating from within the Greenland ice sheet (Lehtinen et al. 2004).

Applied to a potentially much broader-band system as proposed above for SKA air shower radio detection, the multi-band triggering would in principle be applied to each cluster locally. If a trigger occurred, it would cause a global broadcast out to stations within a several $\mathrm{km}$ radius of the triggered cluster, interrogating these other stations to see if they also triggered., When enough stations trigger to justify it, a global trigger would be initiated and all of the stations within the affected distance (including appropriate margin to establish the boundaries of the affected area) would save their buffered data.

The design implications for such a system clearly favor the phase aperture array concept for the SKA, with a low frequency cutoff at the lower end of the VHF band. In many ways the concept of a Low Frequency Array (LOFAR) is perhaps best matched to air shower radio detection, and insight can be gained toward adaptation of the SKA possibilities by considering the adaptation required for a LOFAR-type array. Studies for such applications with LOFAR have been recently published (Falcke \& Gorham 2003, Huege $\&$ Falcke 2002, 2003), and the results are quite promising.

\subsection{Neutrino detection}

In contrast to the problem of air shower radio detection with the SKA, which is driven by the fact that there is only one clear mechanism for detection, neutrino detection with the SKA may be pursued on several fronts. The scientific motivations for both neutrino and air shower detection from $\mathrm{EeV}$ to $\mathrm{ZeV}$ energies are closely related, and neutrino detection at these energies will provide highly complementary information to our current incomplete knowledge of the sources and propagation of the highest energy cosmic rays.

To date, no cosmic high energy $(\geq 1 \mathrm{GeV})$ neutrinos have been detected from any source. The AMANDA detector at Amundsen station, Antarctica, has detected cosmic-ray secondary neutrinos up to $\sim 100 \mathrm{TeV}$ energies, but these arise from interactions of garden-variety $\sim \mathrm{PeV}$ cosmic rays in the Earth's atmosphere.

For this reason, the discussion of neutrino detection must be more broad in scope, since we do not yet know which detection channels might lead to methods with sufficient sensitivity to see fluxes of neutrinos over the entire range of $10^{10-23} \mathrm{eV}$ where they are expected but so far unobserved. This section is therefore more speculative with regard to possible techniques, but appropriate to the high level of scientific interest in neutrino detection.

\subsubsection{Neutrino interactions in the Earth}

At energies of about $1 \mathrm{PeV}$, the earth becomes opaque to neutrinos at the nadir. For higher energies, the angular region of opacity grows from around the nadir till at $\mathrm{EeV}$ energies, neutrinos can only arrive from within a few degrees below the horizon. The interaction length at these energies is of order $1000 \mathrm{~km}$ in water, so such neutrinos have a significant probability of interacting along a $\sim 100 \mathrm{~km}$ chord. If the interaction takes place within $\sim 10 \mathrm{~m}$ of the surface of rock or dry sand or soil, the resulting cascade will produce coherent Cherenkov radiation up to microwave frequencies. Thus, for example, since arrays are often sited with mountains or ridges near the horizon, the entire near-surface volume of the mountain range becomes a neutrino target, and events can originate anywhere along its surface. The flux density expected for such events (cf. Saltzberg et al. 2001) is

$$
\begin{aligned}
S_{\nu} \simeq 12 \operatorname{MJy}\left(\frac{R}{1 \mathrm{~km}}\right)^{-2} \times \\
\\
\quad\left(\frac{E_{c}}{10^{18} \mathrm{eV}}\right)\left(\frac{\nu}{200 \mathrm{MHz}}\right)^{2}
\end{aligned}
$$


where $E_{c}$ is the cascade energy and $R$ the distance to the cascade. ${ }^{2}$ The Cherenkov process weights these events strongly toward the higher frequencies, though events that originate deeper in the ground will have their spectrum flattened by the typical $\nu^{-1}$ behavior of the loss tangent of the material.

A similar process leads to coherent transition radiation (TR; cf. Takahashi et al. 1994) from the charge excess of the shower, if the cascade breaks through the local surface. TR has spectral properties that make it more favorable for an array at lower frequencies: it produces equal power per unit bandwidth across the coherence region. The resulting flux density for a neutrino cascade breaking the surface near the array array, observed at an angle of within $\sim 10^{\circ}$ from the cascade axis, is (cf. Gorham et al. 2000):

$$
\begin{aligned}
S_{\nu, T R}\left(\theta \leq 10^{\circ}\right) \simeq 2 \mathrm{MJy}\left(\frac{R}{1 \mathrm{~km}}\right)^{-2} \times \\
\left(\frac{E_{c}}{10^{18} \mathrm{eV}}\right)^{2} .
\end{aligned}
$$

The implication here is that, if an array can retain some response from the antennas to near-horizon fluxes, the payoff may be a significant sensitivity to neutrino events in an energy regime of great interest around $1 \mathrm{EeV}$, or even significantly below this energy depending on the method of triggering.

\subsubsection{Neutrino interactions in the atmo- sphere}

Neutrinos can themselves also produce air showers. The primary difference between these and cosmic-ray-induced air showers is that their origin, or first-interaction point, can be anywhere in the air column, with an equal probability of interaction at any column depth. Neutrino air

\footnotetext{
${ }^{2}$ Note that in this case the neutrino energy is not necessarily equal to the cascade energy $E_{c}$, because for the typical deep-inelastic scattering interactions that occur for $\mathrm{EeV}$ neutrinos, only about $20 \%$ of the energy is put into the cascade, while the balance is carried off by a lepton. For electron neutrinos, the electron will rapidly interact and add its energy to the shower, but for muon or tau neutrinos, this lepton will generally escape undetected (although the tau lepton will itself decay within a few tens of $\mathrm{km}$ at $1 \mathrm{EeV})$.
}

showers can even be locally up-going at modest angles, subject to the earth-shadowing effects mentioned above.

Detection of such events is identical to detection of cosmic-ray-induced air showers, except for the fact that sensitivity to events from near the horizon is desirable, since these will be most easily distinguished from cosmic-ray-induced events. Beyond a zenith angle of $\sim 70^{\circ}$ cosmic-ray radio events will be more rare, and those that are detected in radio will be distant. The column depth of the atmosphere rises by a factor of 30 from zenith to horizon; thus cosmic ray induced air showers have their maxima many kilometers away at high zenith angles. Neutrino showers in contrast may appear close by, even at large zenith angles.

Of particular interest is the possibility of observing "double-bang" (Learned \& Pakvasa 1995) tau neutrino events. In these events, a $\nu_{\tau}$ interacts first, producing a near-horizontal air shower from a deep-inelastic hadronic scattering interaction. The tau lepton escapes with of order $80 \%$ of the neutrino energy, and then propagates an average distance of $50 E_{\tau} /\left(10^{18} \mathrm{eV}\right) \mathrm{km}$ before decaying and producing (in most cases) another shower of comparable energy to the first. Detection of both cascades within the boundaries of a surface radio array would provide a unique signature of such events. And in light of the recent neutrino results indicating $\nu_{\mu} \rightarrow \nu_{\tau}$ oscillations, it is likely that neutrinos from astrophysically distant sources would be maximally mixed, leading to a significant rate of $\nu_{\tau}$ events.

\subsubsection{Neutrino interactions in the lunar regolith}

There is an analogous process to the earthsurface layer cascades mentioned above which can take place in the lunar surface material (the regolith). In this case the cascade takes place as the neutrino nears its exit point on the moon after having traversed a chord through the lunar limb. This process, first suggested by Dagkesamansky \& Zheleznykh (1989) is the basis of several searches for diffuse neutrino fluxes at energies of $\sim 10^{20} \mathrm{eV}$ (Hankins et al. 1996; Gorham et al. 1999, 2001, Gorham et al. 2003) using 
large radio telescopes at microwave frequencies. Based on the simulations for these experiments (Alvarez Mũniz \& Zas 1997a, 1997b; Zas, Halzen \& Stanev 1992) and confirmation through several accelerator measurements (Gorham et al. 2000 ; Saltzberg et al. 2001), the expected flux density from such an event at about 1 attenuation-length depth in the regolith can be roughly estimated as

$$
S_{\nu}=50 \mathrm{Jy}\left(\frac{E_{c}}{10^{20} \mathrm{eV}}\right)\left(\frac{\nu}{200 \mathrm{MHz}}\right)^{2} \text {. }
$$

Note here that the flux density is far lower than for air shower events, but the two should not be compared, since the lunar regolith events are coherent over $\sim$ degree angular scales, corresponding to several thousand km at the Earth's surface. They also originate from a small, known angular region of the sky (the surface of the moon). Thus their detectability depends on the sensitivity of the synthesized beam, and on the ability of the system to trigger on band-limited pulses.

Transition radiation events may also be detectable in a similar manner, as noted above. For TR from events that break the lunar surface, the resulting pulse differs from a Cherenkov pulse because it is flat-spectrum. Because TR is strongly forward beamed compared to the Cherenkov radiation from the moon, we estimate that the maximum flux density for this case, at an angle of $\sim 1.5^{\circ}$ from the cascade axis, is about a factor of 20 higher than at $\sim 10^{\circ}$. At earth the implied flux density for LOFAR is:

$S_{\max , T R}\left(\theta \simeq 1.5^{\circ}\right) \simeq 40 \mathrm{Jy}\left(\frac{E_{c}}{10^{20} \mathrm{eV}}\right)^{2}$

Although this channel does not provide a higher flux density than the Cherenkov process, it is a flat spectrum process that may in some cases provide more integrated flux across a given band.

These pulses are essentially completely bandlimited prior to their entry into the ionosphere, with intrinsic width of order 0.2 ns. Dispersion delay in the ionosphere will of course significantly impact the shape of any pulse of lunar origin. This will limit the coherence bandwidth for a VHF system. The dominant quadratic part of the dispersion gives an overall delay

$\tau_{\text {ion }}=1.34 \times 10^{-7} \frac{N_{e}}{\nu^{2}}$

where $\tau_{\text {ion }}$ is the delay in seconds at frequency $\nu$ (in $\mathrm{Hz}$ ) for ionospheric column density $N_{e}$ in electrons per $\mathrm{m}^{2}$. For typical nighttime values of $N_{e} \sim 10^{17} \mathrm{~m}^{-2}$ the zenith delay at $200 \mathrm{MHz}$ is $330 \mathrm{~ns}$, and the differential dispersion is of order $3 \mathrm{~ns}$ per $\mathrm{MHz}$, increasing at lower frequencies as $\nu^{-3}$. For bandwidths up to even several tens of $\mathrm{MHz}$ for zenith observations, and perhaps a few $\mathrm{MHz}$ at low elevations, the pulses should remain band-limited. However, coherent de-dispersion will be necessary to accurately reconstruct the broad-band pulse structure.

Although the problem of coherent dedispersion is a difficult one, a system operating in the $0.2-1 \mathrm{GHz}$ range may have an edge in sensitivity over systems operating at higher frequencies, under conditions where the intrinsic neutrino spectra are very hard. This is due to the fact that the loss tangent of the lunar surface material is relatively constant with frequency (Olhoeft and Strangway 1976), and thus the attenuation length increases inversely with frequency. This means that a lower frequency array may probe a much larger effective volume of mass than the higher frequencies can. At $200 \mathrm{MHz}$, the RF attenuation length should be of order $50 \mathrm{~m}$ or more, compared to 5-7 $\mathrm{m}$ at $2 \mathrm{GHz}$. When this larger effective volume is coupled with the larger acceptance solid angle afforded by the broader RF beam of the low-frequency Cherenkov emission, the net improvement in neutrino aperture could well compensate the loss of sensitivity at lower energies by a large margin.

It is also worth noting here that these lunar regolith observations are distinct from other methods in high energy particle detection, in that they do require the array to track an astronomical target, and can and will make use of the synthetic beam of the entire array. This is because, although the sub-array elements should be used for the detection since they will have a beam that covers the entire moon, the Cherenkov beam pattern from an event of lunar origin covers an area of several thousand $\mathrm{km}$ wide at earth, and is thus 
broad enough to trigger the entire array. Postanalysis of such events can then localize them to a few $\mathrm{km}$ at $100 \mathrm{MHz}$ and about $200 \mathrm{~m}$ at $1.4 \mathrm{GHz}$ on the surface of the moon, providing opportunities for more detailed reconstruction of the event geometry. If, as expected, a high resolution $(5 \mathrm{~m}) 3 \mathrm{D}$ cartographic map of the moon will be produced by the Terrain Mapping Camera of India's Chandrayaan-1 lunar mission due for launch in 2008 [www.isro.org/chandrayaan1 /announcement.htm] becomes available, this may be used to determine the local gradient and roughness of the surface near the position of cascade exit. We anticipate that this information, together with the polarisation angle and its frequency dependence, both of which can be measured by the SKA, may enable neutrino direction reconstruction to an "event arc" on the sky of thickness a few degrees to be routinely achieved on an event-by-event basis.

It is in the neutrino energy range most sensitive to UHECR origin at $10^{9}-10^{13} \mathrm{GeV}$ that the SKA may have the greatest impact by using the lunar Cherenkov technique. So far no UHE neutrinos have been detected and the current observational limits are shown in Fig. 17.

In the $10^{11}-10^{13} \mathrm{GeV}$ range the Goldstone Lunar Ultra-high Energy (GLUE) neutrino experiment (Gorham et al. 2003) has the best limit. Other planned experiments in this energy range such as SALSA (Gorham et al. 2002) and ANITA (Barwick et al. 2003) will lower these limits and hopefully detect neutrinos. The lunar Cherenkov technique used in the GLUE experiment was pioneered by Hankins, Ekers \& O'Sullivan (1996) using the Parkes $64 \mathrm{~m}$ radio telescope. The GLUE experiment used two dishes of the Goldstone Deep Space Tracking Network for 120 hours to look for Cherenkov radio emission from neutrinoinduced cascades in lunar regolith.

By scaling relationships given by Gorham et al. (2000) and Alvarez-Muniz \& Zas (2001) describing the electric field strength at the radio telescopes expected for a given cascade energy deposited in the regolith (see also Beresnyak 2003), and comparing the proposed technical specifications of the SKA (assuming $1 \mathrm{GHz}$ frequency will be used, its higher bandwidth and larger tele-

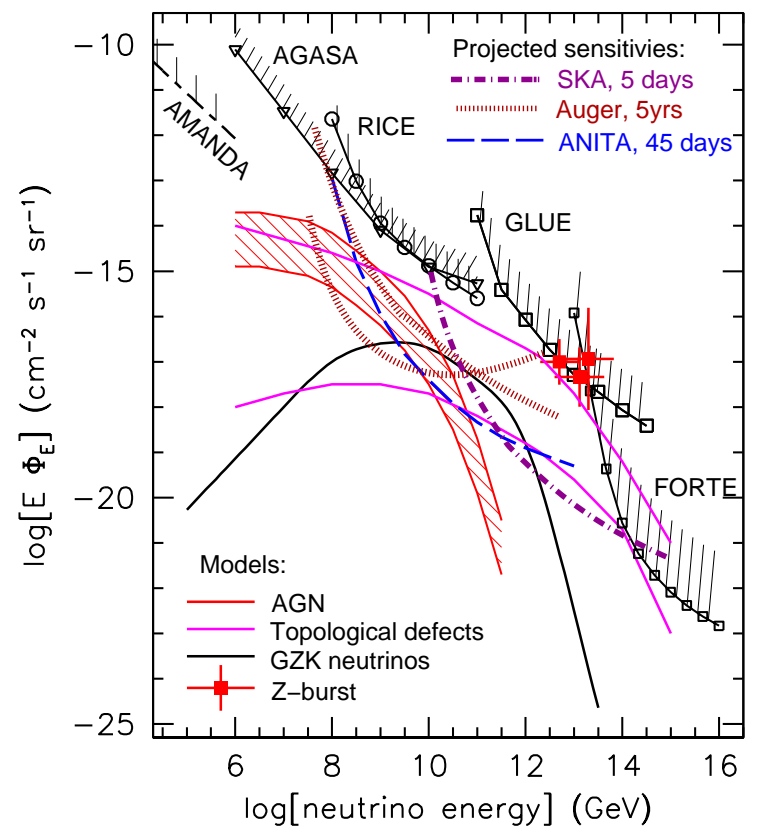

Figure 17. Black solid curves with points show existing limits on diffuse neutrino intensity (for references see Gorham et al. 2003, from which this plot is adapted). Thick, dark red dotted curves show the expected sensitivity of Auger Observatory to $\nu_{\mu}$ and $\nu_{e}$ (top), and $\nu_{\tau}$ (with no deepinelastic-scattering losses, bottom) in 5 years of observations. The magenta dash-dot curve labelled SKA gives an estimates of the expected sensitivity of the SKA as a neutrino observatories for $120 \mathrm{~h}$ of lunar observations. ANITA is an Antarctic Long duration balloon mission due to fly in 2006; this estimate is for the full planned 3 flights by 2009. Other curves bracket AGN predictions, two TD models, and an estimate of the maximal GZK neutrino flux.

scope field of view and collecting area) with those of the telescopes used in the GLUE experiment, one expects that for comparable lunar observing time with the SKA the threshold will be reduced to $2 \times 10^{10} \mathrm{GeV}$ and the sensitivity will be improved by a factor of about 2000 (dot-dash curve 
"SKA" in Fig. 177, making it potentially the most sensitive UHE neutrino observatory in the future for covering a large part of the important energy range $10^{9}-10^{14} \mathrm{GeV}$.

\section{Summary and outlook}

In the next several years giant air shower detectors will investigate the spectrum, composition and anisotropy of the UHECR, i.e. those with energies above $10^{10} \mathrm{GeV}$, in an attempt to determine their origin. Because of its large collecting area, the use of the SKA to directly observe coherent geosynchrotron radio emission from cosmic ray air showers has the potential to make a significant impact in this field. However, cosmic rays are deflected by magnetic fields and do not point back directly to their sources, and above $\sim 10^{11} \mathrm{GeV}$ UHECR suffer severe energy losses on interacting with CMBR photons, limiting their range to tens of $\mathrm{Mpc}$ from their sources. Hence studies of UHECR alone will probably be insufficient to tie down their sources and whether they are accelerated or result from the decay of massive relic particles or emission by topological defects.

UHE neutrinos are the key to determining the origin of these UHECR. This subject is of great importance to our understanding of the Universe as it impacts on our knowledge of dark matter, gravity, and high energy particle interactions. Direct radio observation by the SKA of air showers due to high energy neutrinos may contribute significantly to high energy neutrino astrophysics, particularly below $10^{10} \mathrm{GeV}$. However, the enormous neutrino collecting area of the Moon, together with the large aperture and excellent angular resolution of the SKA make UHE neutrino astrophysics using the lunar Cherenkov technique potentially the best approach for tying down the origin of the very highest energy particles in nature.

The signal coincidence requirement between antennas and the nanosecond duration signal experimental procedures are significantly different from those in normal radio astronomy, and must be taken into account together with the most appropriate signal processing technique for multiple antennas in the design of the SKA if it is to be used for lunar UHE neutrino observations and take a leading role in neutrino astronomy at the highest energies.

Acknowledgement: We thank T. Huege for useful comments on the document.

\section{References}

[1] Abbasi, R. U., et al. 2004, Physical Review Letters, 92, 151101

[2] Agnetta, G. et al. 1997, Astropart. Physics, 6,301

[3] Allan, H.R., Neat, K.P., Jones, J.K. 1967, Nature, 215, 267

[4] Allan, H.R., Clay, R.W., Jones, J.K. 1970, Nature, 227, 1116

[5] Allan, H.R. 1971, Prog. in Elem. Part. and Cos. Ray Phys., ed. J. G. Wilson and S. A. Wouthuysen, (N. Holland Publ. Co.), Vol. 10,171

[6] Alvarez-Muñiz, J., \& Zas, E. 1997a, Phys. Lett. B, 411, 218; also astro-ph/9806098

[7] Alvarez-Muñiz, J., \& Zas, E. 1997b, Proc. XXVth ICRC, ed. M.S. Potgeiter et al. vol. 7,309

[8] Alvarez-Muniz, J., Zas, E. 2001, AIP Conf. Proc. 579, pp 128-138

[9] Alvarez-Muniz, J., Marques, E., Vazquez, R.A., Zas, E. 2003, Phys. Rev., D68, 043001

[10] Arons, J. 2003, ApJ, 589, 871

[11] Askar'yan, G.A. 1962, Sov. Phys. JETP, 14 441; 1965, ibid 48, 988

[12] Barker,H.R., Neat, K.P., Jones, J.K. 1967, Nature 215, 267

[13] Barwick, S.W. et al. 2003, Proc. SPIE, 4858, 265

[14] Beresnyak, A.R. 2003, astro-ph/0310295

[15] Bird, D.J. et al. 1995, ApJ, 441, 144 
[16] Bourdeau, M.F., J.N.Capdevielle, J.N. \& Procureur,J. 1980, J. Phys. G, 6, 901

[17] Colgate, S.A., 1967, J. Geophys. Res., 72, 4869

[18] Dagkesamansky, R.D. \& Zheleznykh, I.M. 1989, Sov. Phys. JETP Lett., 50, 233

[19] Dova, M.T., Fanchiotti, H., Garcia-Canal, C. et al. 1999, Proc. 26th ICRC, Salt Lake City Utah 1999, Ed. D. Kieda, M. Salamon, B. Dingus, Vol. 1, p. 514 (HE.2.5.34), http://krusty.physics.utah.edu/ icrc1999

[20] Engel, R. Seckel, D. Stanev, T. 2001, Phys. Rev. D64, 093010

[21] Falcke, H. \& Gorham, P. 2003, Astropart. Phys., 19, 477

[22] Gorham, P.W., Liewer, K.M., and Naudet, C.J. 1999, Proc. 26th ICRC, Salt Lake City, Utah, HE.6.3.15

[23] Gorham, P.W, Saltzberg, D.P., Schoessow, P. et al. 2000, Phys. Rev., E 62, 8590

[24] Gorham, P. et al. 2002, Nucl. Instrum. Meth., A490, 476

[25] Gorham, P. W., Hebert, C. L., Liewer, K. M., Naudet, C. J., Saltzberg, D., \& Williams, D. 2004, Physical Review Letters, 93, 041101

[26] Gorham, P. W., Liewer, K. M., Naudet, C. J., Saltzberg, D. P., \& Williams, D. 2001, AIP Conf. Proc. 579: Radio Detection of High Energy Particles, 579, 177

[27] Gorham, P.W. et al. 2004, in preparation for PRD.

[28] de Gouvela Dal Pino, E.M., Lazarian, A. 2001, ApJ, 560, 358

[29] Green, K., Rosner, J.L.D., Suprun, A. \& Wilkerson, J.F. 2003, Nucl. Instr. Meth. A, 498, 256

[30] Greisen, K. 1956, Prog. Cosmic Ray Physics., Suppl. 3.
[31] Greisen, K. 1966, Phys. Rev. Lett., 16, 748

[32] Hankins, T.H., Ekers, R.D., O'Sullivan, J.D. 1996, MNRAS, 283, 1027

[33] Haswell, C.A., Tajima, T., \& Sakai, J.-L. 1992, ApJ, 401, 495

[34] Hillas, A.M. 1984, Ann. Rev. Astron. Astrophys., 22, 425

[35] Horneffer, A., et al., 2003, in: "Proceedings of the 28th ICRC, Tsukuba, Japan", Vol. HE 1.5.15, p. 969

[36] Horneffer, A., et al., 2004, in: "Astronomical Telescopes and Instrumentation 2004: Gravitational Wave and Particle Astrophysics detectors", Proc. SPIE, Vol. 550021

[37] Hough, J.H., Prescott, J.R. 1970, Nature 227, 591

[38] Hough, J.H. 1973, J. Phys. A, 6, 892

[39] Huege, T., \& Falcke, H., Proc. of the 6th European VLBI Network Symposium, Ros E., Porcas R.W., Lobanov, A.P., \& Zensus, J.A. (eds.), MPIfR, Bonn, Germany (2002), p. 25

[40] Huege, T., \& Falcke, H., Astron. Astrophys., 2003, 412, 19

[41] Huege, T., \& Falcke, H., Astron. Astrophys., 2004, subm.

[42] Jackson, J.D. 1999, Classical Electrodynamics, 3rd Edition (Wiley), p. 664.

[43] Jones, F.C., \& Ellison, D.C. 1991, Space Sci. Rev., 58, 259

[44] Kahn, F.D., Lerche, I. 1966, Proc. Roy. Soc. London, A-289, 206

[45] Kamata, K., \& Nishimura, J. 1958, Prog. Theor. Phys. Suppl. 6, 93

[46] Learned, J. G., \& Pakvasa, S., 1995, Astropart. Phys. 3, 267. 
[47] Lehtinen, N.G., Gorham, P.W., Jacobson, A.R. \& Roussel-Dupre, R.A. 2004, Phys. Rev., D69, 013008

[48] Linsley, J. 1986, J. Phys. G, 12, 51

[49] Litwin, C., Rosner, R. 2001, Phys. Rev. Lett., 86, 4745

[50] Medvedev, M.V. 2003, Phy. Rev. E, 67, 045401

[51] Mücke, A., Protheroe, R.J., Engel, R., Rachen, J.P., \& Stanev, T. 2003, Astropart. Phys., 18, 593

[52] Nagano, M., \& Watson, A.A. 2000, Rev. Mod. Phys., 72, 3, 689

[53] Olhoeft, G.R. \& Strangway, D.W. 1975, Earth Plan. Sci. Lett., 24, 394

[54] Pelletier, G., Kersalé, E. 2000, Astron. Astrophys., 361, 788

[55] Prah, J. H. 1971, M.Phil. thesis, University of London

[56] Protheroe, R.J. 2000, in Topics in cosmicray astrophysics, ed. M.A. DuVernois, Nova Science Publishing: New York, pp 258-298

[57] Protheroe, R.J., Donea, A.-C., Reimer, A. 2003, Astropart. Phys., 19, 559

[58] Protheroe, R.J. \& Clay, R.W. 2004, Publ. Astron. Soc. Australia, 21, 1

[59] Protheroe, R.J. 2004, Astropart. Phys. 21, 415

[60] Rybicki, G.B., Lightman A.P. 1979, Radiative Processes in Astrophysics, (John Wiley \& Sons, New York)
[61] Saltzberg, D., Gorham, P., Walz, D. et al. 2001, Phys. Rev. Lett., 86, 2802; hep-ex/0011001

[62] Schieler, H. and the KASCADE and LOPES collaborations, 2003, in: Particle Astrophysics Instrumentation, Peter W. Gorham. (ed.), Proceedings of the SPIE, Volume 4858, pp. 41-55

[63] Sorrell, W.H. 1987, ApJ, 323, 647

[64] Spencer, R.E. 1969, Nature 222, 460

[65] Stanev, T., Engel, R., Mücke, A., Protheroe, R.J., \& Rachen, J.P. 2000, Phys. Rev. D, 62, 093005

[66] Suprun, D., Gorham, P. \& Rosner, J. 2003, Astropart. Phys., 20, 157

[67] Takahashi, T. et al., 1994, Phys. Rev. E, 50,4041

[68] Takeda, M., et al. 2003, Astropart. Phys., 19,447

[69] Vernov, S.N. et al. 1968, Can. J. Phys. 46, 241

[70] Wheeler, J.A., \& Feynman, R.P. 1949, Rev. Mod. Phys., 21, 425

[71] Yamamoto, T., Mase, K., Takeda, M., Sakaki, N., Teshima, M. 2004, Astropart. Phys., 20, 405

[72] Zas, E., Halzen, F., Stanev, T. 1992, Phys. Rev. D, 45, 362

[73] Zatsepin, G.T. \& Kuzmin, V.A. 1966, Sov. Phys. JETP Lett., 4, 78 